\title{
Amyloid precursor protein-mediated endocytic pathway disruption induces axonal dysfunction and neurodegeneration
}

\author{
Wei Xu, ${ }^{1,2}$ April M. Weissmiller, ${ }^{2}$ Joseph A. White II, ${ }^{3}$ Fang Fang, ${ }^{1,2}$ Xinyi Wang, ${ }^{1}$ Yiwen Wu, ${ }^{1}$ Matthew L. Pearn, ${ }^{4,5}$ Xiaobei Zhao, ${ }^{2}$ \\ Mariko Sawa, ${ }^{2}$ Shengdi Chen, ${ }^{1}$ Shermali Gunawardena, ${ }^{3}$ Jianqing Ding, ${ }^{1}$ William C. Mobley, ${ }^{2}$ and Chengbiao Wu ${ }^{2}$ \\ 'Department of Neurology and Institute of Neurology, Ruijin Hospital, Shanghai Jiao Tong University School of Medicine, Shanghai, China. ${ }^{2}$ Department of Neurosciences, UCSD, La Jolla, California, USA \\ ${ }^{3}$ Department of Biological Sciences, The State University of New York at Buffalo, Buffalo, New York, USA. ${ }^{4}$ Department of Anesthesiology, UCSD, La Jolla, California, USA. ${ }^{5}$ VA San Diego Healthcare System, \\ San Diego, California, USA.
}

\begin{abstract}
The endosome/lysosome pathway is disrupted early in the course of both Alzheimer's disease (AD) and Down syndrome (DS); however, it is not clear how dysfunction in this pathway influences the development of these diseases. Herein, we explored the cellular and molecular mechanisms by which endosomal dysfunction contributes to the pathogenesis of $A D$ and $D S$. We determined that full-length amyloid precursor protein (APP) and its $\beta$ - $C$-terminal fragment ( $\beta$-CTF) act though increased activation of Rab5 to cause enlargement of early endosomes and to disrupt retrograde axonal trafficking of nerve growth factor (NGF) signals. The functional impacts of APP and its various products were investigated in PC12 cells, cultured rat basal forebrain cholinergic neurons (BFCNs), and BFCNs from a mouse model of DS. We found that the full-length wild-type APP (APP'T) and $\beta$-CTF both induced endosomal enlargement and disrupted NGF signaling and axonal trafficking. $\beta$-CTF alone induced atrophy of BFCNs that was rescued by the dominant-negative Rab5 mutant, Rab5 ${ }^{\mathrm{S} 34 \mathrm{~N}}$. Moreover, expression of a dominant-negative Rab5 construct markedly reduced APP-induced axonal blockage in Drosophila. Therefore, increased APP and/or $\beta$-CTF impact the endocytic pathway to disrupt NGF trafficking and signaling, resulting in trophic deficits in BFCNs. Our data strongly support the emerging concept that dysregulation of Rab5 activity contributes importantly to early pathogenesis of $A D$ and $D S$.
\end{abstract}

\section{Introduction}

Alzheimer's disease (AD) is a progressive neurodegenerative disorder causing memory loss and cognitive decline unless disease-modifying treatments are applied (1-6). The classical neuropathological hallmarks, $\mathrm{A} \beta$-amyloid-containing plaques and tau-containing neurofibrillary tangles, have stimulated studies to define mechanisms of neurodegeneration (1-3, 6-12) that have guided clinical trials (13-16).

Dysfunction of the endocytic system, manifested by enlargement of $\mathrm{Rab}^{+}$early endosomes, is another important neuropathological marker in AD (17-21). Rab5, a small GTPase, regulates endocytosis and intracellular trafficking $(22,23)$. By cycling between an active form (GTP-bound) and an inactive state (GDP-bound), Rab5 dictates endocytosis, trafficking, and sorting of surface cargoes $(18,19,22,23)$. In $\mathrm{AD}$, abnormal enlargement of Rab5 ${ }^{+}$early endosomes is not only characteristic, it occurs early on; it was observed in individuals with sporadic $\mathrm{AD}$ (24). Interestingly, endosomal pathology was detected in brain regions free of $A \beta$ or tau pathology $(24,25)$. In people with Down syndrome (DS), enlarged $\mathrm{Rab}^{+}$endosomes were also observed occurring as early as in the fetal period (26-28). Early endosome abnormalities precede not only the onset of

Conflict of interest: The authors have declared that no conflict of interest exists. Submitted: April 20, 2015; Accepted: February 24, 2016.

Reference information: / Clin Invest. 2016;126(5):1815-1833. doi:10.1172/JCI82409. dementia but also the emergence of plaques and tangles. Therefore, dysregulation of Rab5 activity and of the endocytic pathway are early features in both $\mathrm{AD}$ and DS.

The mechanism(s) leading to endosomal changes in $\mathrm{AD}$ and DS remain undefined. Studies have shown that increased gene dose for the amyloid precursor protein (APP), triplicated in DS, is linked to endosomal abnormalities in DS $(19,26,28,29)$. APP is sequentially cleaved by either $\beta$ - or $\alpha$-secretase to produce the corresponding C-terminal fragments: $\beta$-CTF (or C99) or $\alpha$-CTF (or C83) (refs. 30, 31, and Supplemental Figure 1A; supplemental material available online with this article; doi:10.1172/JCI82409DS1). Cleavage of the $\beta$-CTF by $\gamma$-secretase yields the APP intracellular domain (AICD) and A $\beta$ peptides; $\gamma$-secretase cleavage of $\alpha$-CTF yields AICD and P3 (refs. 32-34 and Supplemental Figure 1A). The toxicity of $A \beta$ peptides $\left(A \beta_{40 / 42}\right)$ has been extensively studied in $A D(1$, $4,5,8)$; the role(s) of full-length APP and/or its CTFs in AD pathogenesis are less well understood (35). Nevertheless, increased APP gene dose in DS, which results in increased levels of the full-length APP and its CTFs, is linked to endosomal pathology $(24,26,28)$. Recent findings for $\beta$-CTF-mediated changes in endosomes in fibroblasts further support that APP is linked to endosomal dysfunction $(19,26,28,29,36)$. However, important questions remain: (a) What APP product(s) cause dysregulation of early endosomes; (b) Which physiological events are impacted, and of these, which might contribute to AD pathogenesis in DS? In the Ts65Dn mouse model of DS, increased App gene dose-mediated enlargement of 
$\mathrm{Rab5}^{+}$endosomes $(26,28,37)$ was correlated with reduced endosomal trafficking and signaling of nerve growth factor (NGF), leading to degeneration of basal forebrain cholinergic neurons (BFCNs) $(28,38)$. Noteworthy was the inverse correlation between APP CTFs and the degree to which transport was reduced (28). Further, expression of either the wild-type human APP or a mutant APP also caused a reduction in NGF transport in Ts65Dn mice (28). Additionally, the $\gamma$-secretase inhibitor (GSI) semagacestat, which eliminates all $\mathrm{A} \beta$ species while increasing APP-CTF levels, has shown severe adverse effect in clinical trials, which is likely associated with APP CTFs in AD patients $(13,14,39,40)$. APP CTFs have since been shown to induce neuronal dysfunction and cognitive deficits in animal models (41-44).

We investigated the mechanism and physiological significance of excessive APP and its CTFs on neuronal function by examining endosomal trafficking of NGF (45-47), critical for BFCNs (48-50). Retrograde axonal trafficking of NGF to BFCN soma is mediated by Rab5 $^{+}$endosomes $(48,49,51,52)$. The selective loss of NGF signals is a prominent feature in both $\mathrm{AD}$ and $\mathrm{DS}$. We demonstrated that fulllength $A P P^{W T}$, a $\beta$-cleavage-resistant mutant (APP $\left.{ }^{\mathrm{M} 596 \mathrm{~V}}\right)$, the familial $\mathrm{AD}$ (FAD) Swedish mutant (APP ${ }^{\mathrm{SWE}}$ ), and $\beta$-CTF all induced Rab5 activation and enlargement of $\mathrm{Rab}^{+}$endosomes. Furthermore, expression of full-length $\mathrm{APP}{ }^{\mathrm{WT}}$ or $\beta$-CTF impaired NGF signaling and retrograde axonal transport, resulting in atrophy of BFCNs. These effects were rescued by expression of a dominant-negative Rab5 mutant both in vitro and in vivo, demonstrating a direct role for increased Rab5 activation in disruption of NGF trafficking and signaling in $\mathrm{AD}$ and $\mathrm{DS}$. Thus, activation of Rab5 is an early neuropathology that may contribute to neurodegeneration in these diseases.

\section{Results}

Rab5 $5^{+}$endosomes were enlarged in cultured BFCNs from Ts65Dn mice. To study the mechanism(s) by which App gene dose impacts the endosomal pathway, we used primary BFCNs from Ts65Dn mice, harboring three copies of the App gene $(28,38,53)$. E18 BFCNs from Ts65Dn mice and $2 \mathrm{~N}$ controls were dissected and cultured in vitro for 7 days (DIV7). Immunostaining was used to confirm the presence of cholinergic, NGF-responsive neurons in the culture with specific antibodies against choline acetyltransferase (ChAT), a marker for cholinergic neurons, and TrkA, the receptor for NGF. The majority of neurons (>90\%) were positive for both markers (Figure 1A). Rab5 $5^{+}$puncta, as stained with a specific antibody, were seen in cell bodies and in processes. In $2 \mathrm{~N}$ neurons, Rab5 $5^{+}$puncta, of various sizes, showed a uniform, smoothly rounded shape (Figure 1B, arrowheads). In Ts65Dn neurons, puncta were larger and their shapes less uniform, frequently appearing as lobular. The average area of puncta was significantly $(P<0.0001)$ increased by approximately $60 \%$ in Ts65Dn as compared with $2 \mathrm{~N}$ neurons (Figure 1C). Histogram analysis showed that Ts65Dn neurons had a significant decrease in the number of the smallest Rab5 ${ }^{+}$puncta $\left(<0.3 \mu \mathrm{m}^{2}\right)$, concomitant with a significant increase in the largest puncta $\left(>0.6 \mu \mathrm{m}^{2}\right)$. In this largest bin, the increase was approximately 4 -fold of that in $2 \mathrm{~N}$ neurons. Thus, $\mathrm{Rab}^{+}$endosomes were markedly altered in Ts65Dn neurons (Figure 1D).

The increase in $\mathrm{Rab5}^{+}$puncta size was likely due to enhanced activation of Rab5, i.e., GTP-Rab5 (22), which induces homotypic fusion of early endosomes, causing their enlargement $(19,22,23$,
$26,54,55)$. We assayed the level of GTP-Rab5 in brains of 12 -monthold Ts65Dn and $2 \mathrm{~N}$ mice following a published protocol (56). As previously reported (28), the level of full-length APP in Ts65Dn was approximately 1.5 -fold than in $2 \mathrm{~N}$ samples (Figure $2 \mathrm{~A}$ ). A significant, approximately $60 \%$ increase in the level of GTP-Rab5 was seen in Ts65Dn as compared with $2 \mathrm{~N}$ samples $(P=0.044)$ (Figure 2, A and B). To test the effect of App gene dose on activated Rab5, we examined $2 \mathrm{~N}$ mouse lines with 2, 1, or 0 copies of the App gene $\left(A p p^{+/+}, A p p^{+/}\right.$, and $\left.A p p^{-/-}\right)$. The levels of the full-length APP protein in embryonic brain tissues, as assayed by immunoblotting, mirrored their respective genotypes (Figure 2C). The level of GTP-Rab5 in $A p p^{-/-}$mice was approximately $70 \%(P=0.002)$ and in $A p p^{+-}$mice approximately $85 \%(P=0.201)$ relative to that in $A p p^{+/+}$mice (Figure 2, C and D). The level of GTP-Rab5 in mouse brain thus correlates inversely with APP gene dose.

We next tested whether the increase in App gene dose in Ts65Dn $\mathrm{BFCNs}$ was responsible for enlargement of Rab5 ${ }^{+}$endosomes (Figure 1). By immunoblotting, the APP siRNA caused an approximately $30 \%$ reduction in the level of full-length APP, as compared with control siRNA (Figure 2E). Rab5 $5^{+}$puncta in BFCNs treated with either the APP siRNA or control siRNA were analyzed (Figure 2, F and G) as in Figure 1. Large, sometimes lobulated Rab5 $5^{+}$puncta were seen in cultures treated with the control siRNA, whereas these structures were typically smaller and rounded in cultures treated with the APP siRNA (Figure $2 \mathrm{G}$ ). Treatment with the APP siRNA significantly reduced the size of $\mathrm{Rab5}^{+}$puncta in Ts65Dn neurons to a value equivalent to that in $2 \mathrm{~N}$ neurons (Figure 2F). Thus, increased App gene dose is necessary for increased Rab5 activation and for early endosome enlargement in Ts65Dn neurons.

Full-length APP and $\beta$-CTF caused enlargement of early endosomes in PC12 cells. To determine how increased APP expression caused an increase in Rab5 activation, we asked which APP product(s) were responsible (Supplemental Figure 1A). We transfected PC12M cells with full-length APP-GFP, C99-GFP ( $\beta$-CTF), C83-GFP ( $\alpha$-CTF), or AICD-GFP and examined endosomes by live cell imaging (Supplemental Figure 1B). Bright foci of $\mathrm{GFP}^{+}$ intracellular structures were present in $\mathrm{PC} 12 \mathrm{M}$ cells that overexpressed APP-GFP or C99-GFP. In contrast, cells expressing C83-GFP or AICD-GFP showed diffuse, hazy signals for GFP, with occasional foci in C83-GFP cells. In APP-GFP and C99-GFP cells, the $\mathrm{GFP}^{+}$intracellular structures were, on average, approximately $2 \mu \mathrm{m}^{2}$ (Supplemental Figure 1E). GFP signals in C83-GFP or AICD-GFP cells contained speckled small puncta within the haze, as well as a small number of larger bright puncta, as seen in cells expressing C99-GFP and APP-GFP (Supplemental Figure 1B). However, the average puncta size in C83-GFP and AICD-GFP cells was approximately 1.2 and $1.3 \mu \mathrm{m}^{2}$, respectively. Thus, overexpressing APP and $\beta-\mathrm{CTF}$, but not $\alpha-\mathrm{CTF}$ or AICD, routinely induced formation of enlarged, bright intracellular structures. We also tested two APP mutants: $\mathrm{APP}^{\mathrm{M} 596 \mathrm{~V}}$ and $\mathrm{APP}^{\mathrm{SWE}}$. $\mathrm{APP}^{\mathrm{M} 596 \mathrm{~V}}$, which abolishes $\beta$-secretase cleavage, prevents production of $\beta$-CTF (57); APP ${ }^{\mathrm{SWE}}$ enhances $\beta$-secretase cleavage to increase the level of $\beta$-CTF (57). Both induced the formation of enlarged intracellular structures (Supplemental Figure 1C).

We examined colocalization of APP or C99 with Rab5 in cotransfection experiments; APP-mCherry with GFP-Rab5 ${ }^{\text {WT }}$ (Figure 3B); C99-GFP with mCherry-Rab5 ${ }^{\mathrm{WT}}$ (Figure 3C); and 
A
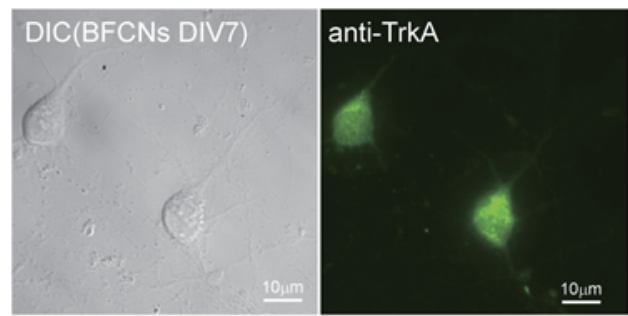

anti-ChAT
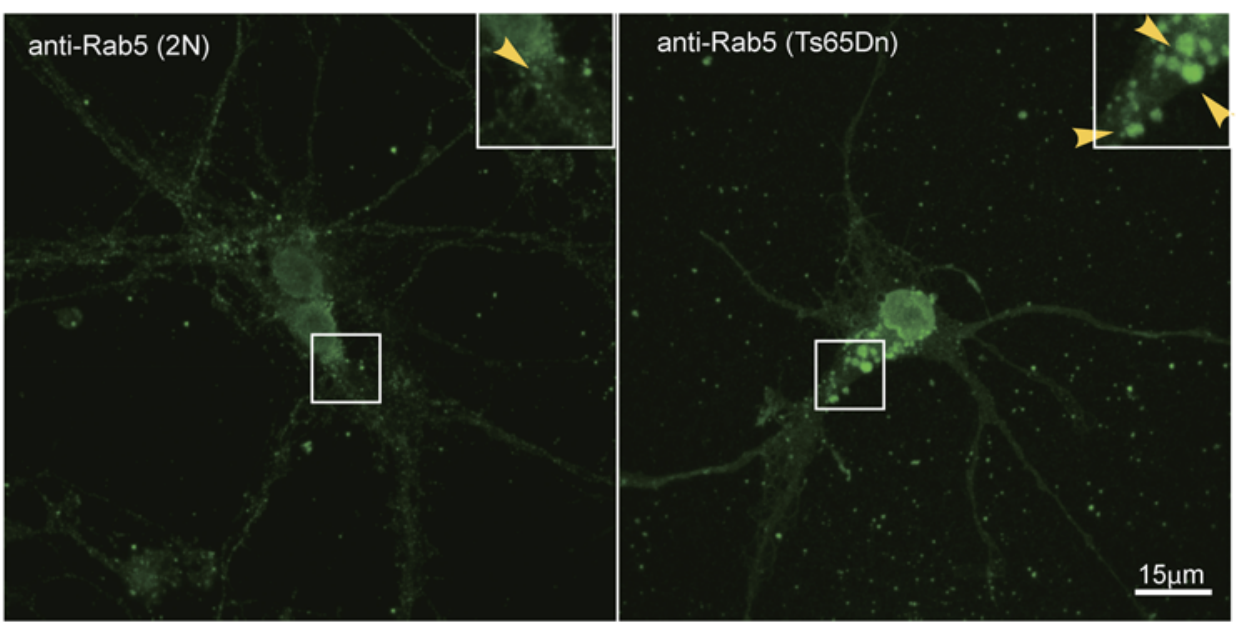

C

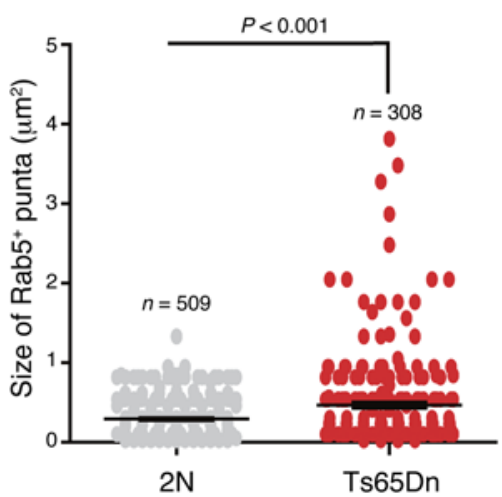

D

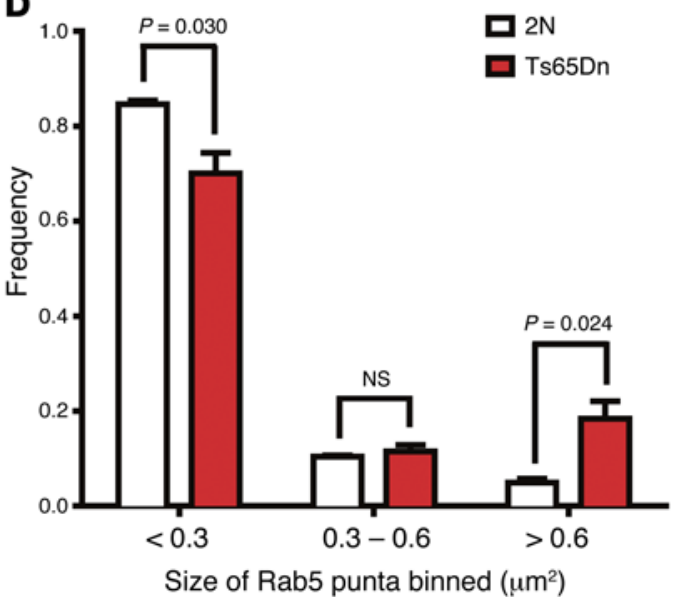

Figure 1. Rab5 ${ }^{+}$early endosomes were enlarged in primary BFCNs of Ts65Dn mice. (A) Representative images of primary BFCNs (DIV7) were costained for the cholinergic neuronal marker ChAT (red) and the NGF receptor TrkA (green). DIC and merged images are also shown. Scale bars: $10 \mu \mathrm{m}$. (B) Representative images are shown for Rab5 staining of BFCNs from Ts65Dn (right) and $2 \mathrm{~N}$ littermates (left). The sizes of Rab5 puncta in BFCNs from Ts65Dn and $2 \mathrm{~N}$ littermates were quantified using ImageJ. Insets: Zoom-in ( $\times 2)$ images of selected areas. Scale bar: $15 \mu \mathrm{m}$. (C) Measurement of Rab5 ${ }^{+}$puncta in B. The average area was $0.468 \mu \mathrm{m}^{2}$ ( $n=308)$ for Ts65Dn, $0.295 \mu \mathrm{m}^{2}(n=$ 509) for $2 \mathrm{~N}$. The measurements were from 3 experiments, with $20-30$ cells analyzed each time. (D) The size distribution of Rab5 $5^{+}$puncta in BFCNs from Ts65Dn mice showed a shift from smaller to larger binned areas in comparison to those from $2 \mathrm{~N}$ littermates. All data represent mean $\pm \operatorname{SEM}(n=3)$, and $P$ values were calculated using Student's $t$ test. n.s., nonsignificant.
C83-GFP with mCherry-Rab5 ${ }^{\mathrm{WT}}$ (Figure 3D). As a control, EGFP was also cotransfected with mCherry-Rab5 ${ }^{\mathrm{WT}}$ (Figure $3 A)$. Cotransfection of mCherry-Rab5 ${ }^{\mathrm{WT}}$ with EGFP yielded small cytoplasmic Rab5 $5^{+}$puncta, with an increase in the number located in the perinuclear region (Figure $3 \mathrm{~A}$ ). $\mathrm{APP}^{+}$puncta were typically circular and quite large, with a bright rim and a dark center (Figure 3B); these structures colocalized with mCherryRab5 $^{\text {WT }}$ (Figure 3B). C99 expression induced large puncta that also colocalized with mCherry-Rab5 ${ }^{\mathrm{WT}}$ (Figure 3C). Though APP and C99 induced very similar changes, the overall extent of colocalization with Rab5 appeared to be greater for C99 (Figure 3, B and C). C83 expression (Figure 3D) did not induce these changes; the size and distribution of early endosomes were similar to the EGFP-expressing control (Figure 3A), except that in these small vesicles, C83 colocalized with mCherry-Rab5 ${ }^{\mathrm{WT}}$ (Figure 3D). Thus, overexpression of APP and C99, but not C83, resulted in enlargement of early endosomes.
Accumulation of $\beta$-CTF (C99) induces synaptic toxicity with a detrimental effect on neuronal function (41-43, 58-60). To explore whether C99-induced changes might be reflected in endosome structure and function, we tested APPSWE-YFP and $\mathrm{APP}^{\mathrm{M} 596 \mathrm{~V}}$-YFP; expression of APP ${ }^{\mathrm{SWE}}$ provided a means to markedly increase C99 (57), while APP ${ }^{\mathrm{M} 596 \mathrm{~V}}$ enabled us examine the effect of full-length APP in the absence of C99 (57). These constructs were coexpressed in PC12 cells with mCherry-Rab5 ${ }^{\mathrm{WT}}$. Cells expressing either $\mathrm{APP}^{\mathrm{SWE}}$ (Figure $4 \mathrm{~A}$ ) or $\mathrm{APP}^{\mathrm{M} 596 \mathrm{~V}}$ (Figure 4B) exhibited enlarged intracellular structures similar to those seen with fulllength APP and C99 (Figure 3, B and C). These large circular structures, albeit to a more dramatic extent in cells expressing $\mathrm{APP}^{\mathrm{M} 596 \mathrm{~V}}$ (Figure 4B), had a bright rim and a dark center, and colocalized extensively with mCherry-Rab5 ${ }^{\mathrm{WT}}$, indicating that full-length APP and C99 act to increase the size of early endosomes.

We predicted that increased Rab5 activation would produce similar effects. Indeed, extremely large vesicular structures were 

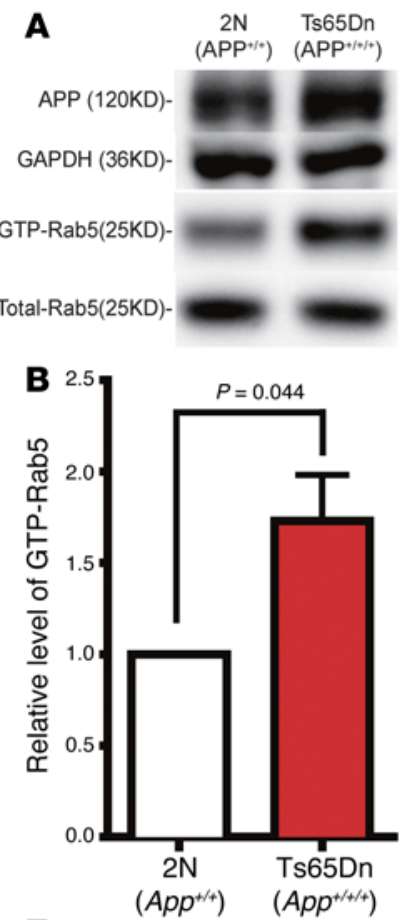

E

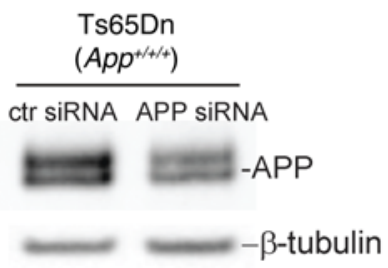

C

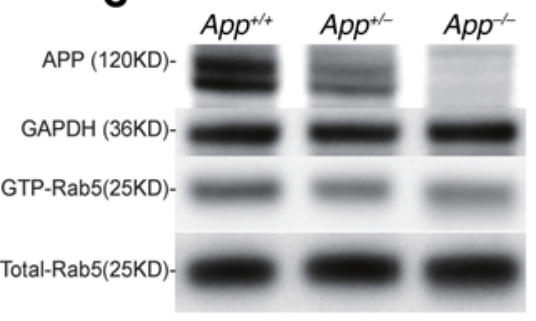

D
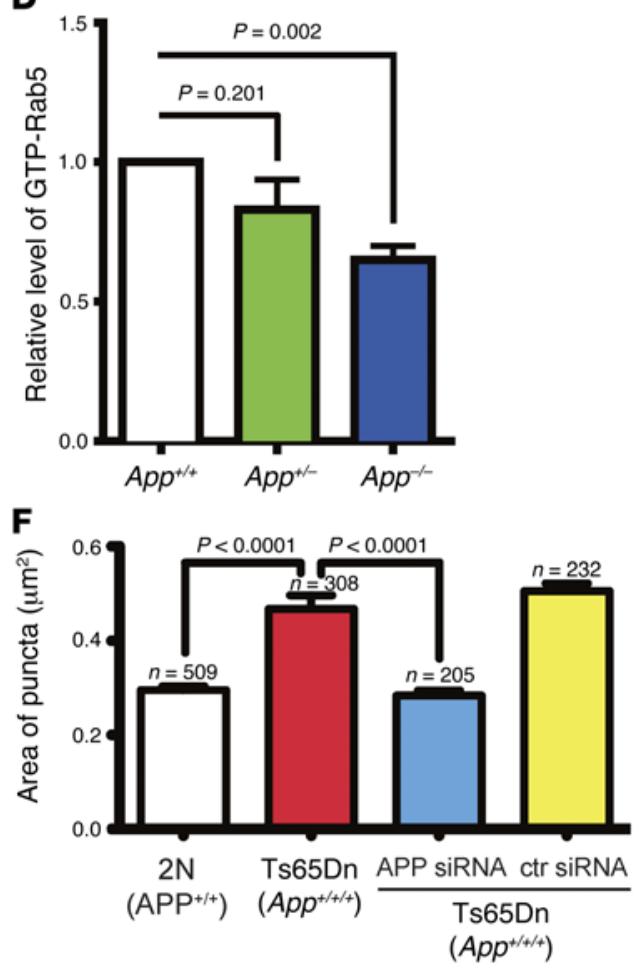

Figure 2. The level of GTP-Rab5 correlated with App gene dose in mouse brain tissues. (A) Protein levels of APP, GAPDH, GTP-Rab5, and total Rab5 in brain homogenates from 12-month-old Ts65Dn and 2N littermates were assayed as described in Methods. (B) The levels of GTP-Rab5 in these samples were quantitated and normalized against internal control, showing a $73 \%$ increase in 12-month-old Ts65Dn samples relative to $2 \mathrm{~N}$ littermates. The level of GTP-Rab5 in $2 \mathrm{~N}$ littermates was set at 1 . (C) The protein level of APP in the brain homogenates from E17 $\mathrm{App}^{+/+}, \mathrm{App}^{+/-}$, and $\mathrm{App}^{-/-}$embryos was detected using an antibody against the C-terminus of APP (top lane). The level of GTP-Rab5 in $\mathrm{App}^{+/+}$mouse brains was set at 1 , with the relative levels shown in $\mathbf{D}$. (E) APP levels were detected in BFCNs of Ts65Dn mice that were treated with control or APP siRNA; $\beta$-tubulin was used as a loading control. (F) The size of Rab5 ${ }^{+}$puncta was significantly reduced by APP siRNA, compared with control siRNA (ctr siRNA). (G) Representative images showing Rab5 staining of BFCNs from Ts65Dn mice with APP knockdown (right) and control siRNA (Scramb., scrambled; left). Insets:

Zoom-in ( $\times 2.5)$ images of the selected areas. Scale bars: $15 \mu \mathrm{m}$. All data represent mean \pm SEM of $\geq 3$ independent experiments, and $P$ values were calculated using Student's $t$ test.

\section{G}

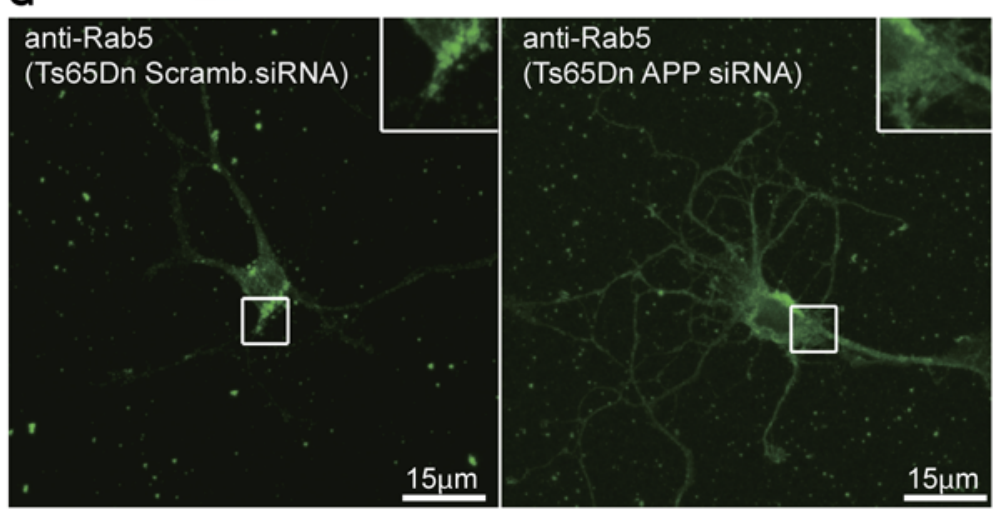

seen within the cytoplasm of the cell expressing either Rabex-5GFP (RAB guanine nucleotide exchange factor 1 for Rab5) (ref. 61 and Figure 4C) or GFP-Rab5 ${ }^{\mathrm{Q} 79 \mathrm{~L}}$, a constitutively active mutant of Rab5 (ref. 62 and Figure 4D). Colocalization with mCherry-Rab5 ${ }^{\text {WT }}$ was readily appreciated in these cells (Figure 4, C and D).

Quantitative analysis confirmed that overexpression of the full-length APP, or two APP mutants (APP ${ }^{\mathrm{M} 596 \mathrm{~V}}, \mathrm{APP}^{\mathrm{SWE}}$ ), or $\mathrm{C} 99$ significantly increased the size of Rab5 ${ }^{+}$puncta (Figure 4, E and F). C83 had no significant impact in these measures (Figure 4, E and $\mathrm{F}$ ), providing further evidence that $\beta-\mathrm{CTF}$ and $\alpha$-CTF have very different effects on $\mathrm{Rab5}^{+}$endosomes. Consistent with reports that altered processing or increased gene dose of APP contributed to enlargement of Rab5 ${ }^{+}$endosomes in $\mathrm{AD}$ and DS $(18,26$, 36 ), these results have shown that both full-length APP and $\beta$-CTF induce enlargement of $\mathrm{Rab5}^{+}$early endosomes, likely through upregulating Rab5 activation.

Full-length APP and C99 induced endosomal enlargement by enhancing activation of Rab5. To determine whether increased expression of full-length APP and C99 enhanced activation of

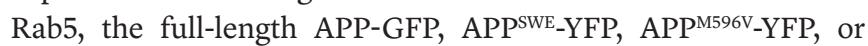




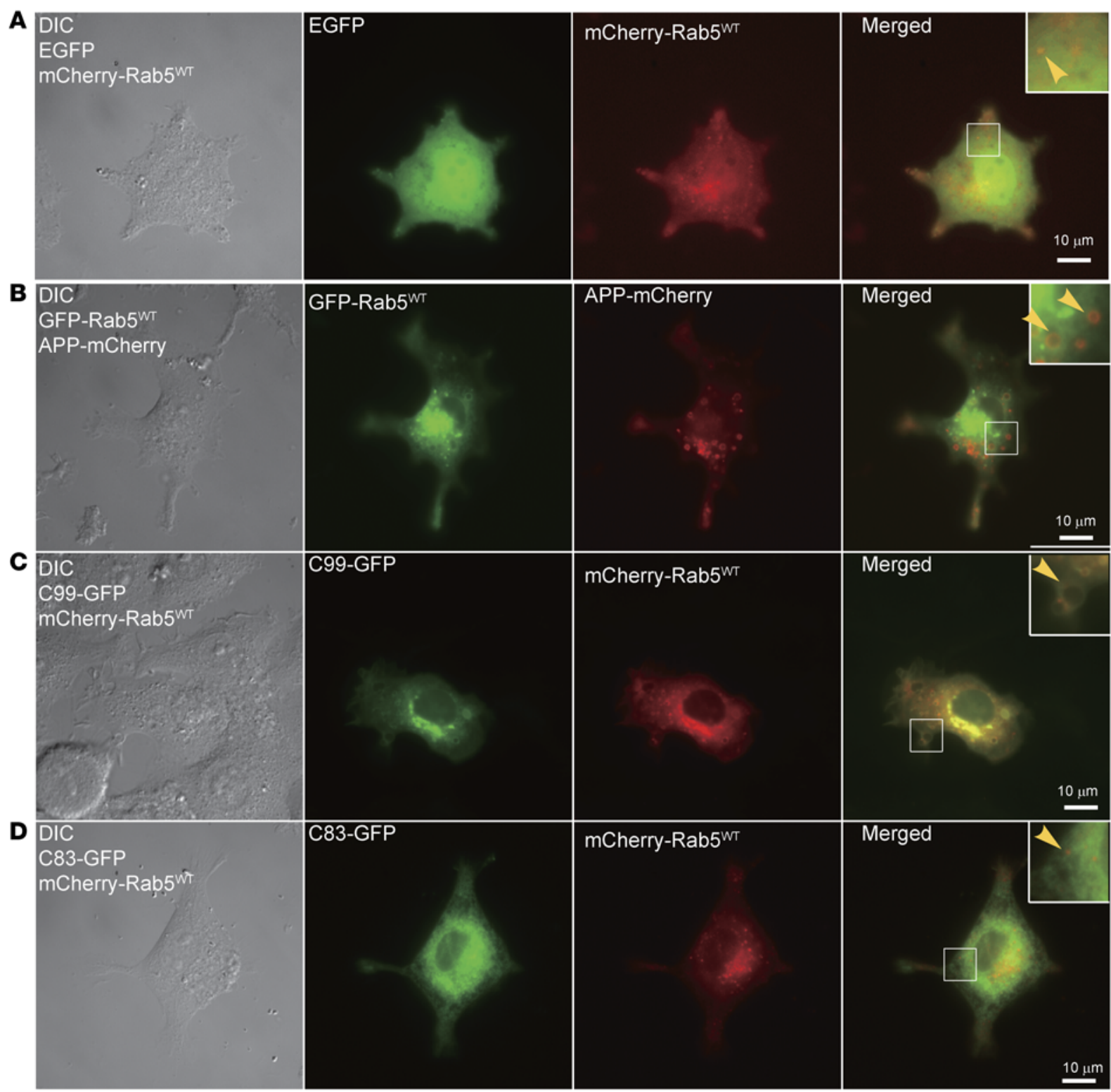

Figure 3. Full-length APP and C99, but not C83, induced the enlargement of Rab5+ endosomes in PC12M cells. PC12M cells were cultured on glass coverslips and cotransfected with the indicated plasmids. Live cell imaging was performed as described in Methods. Images of DIC, FITC, and Texas Red channels were collected, and representative images are shown. Images for cotransfection of mCherry-Rab5WT with EGFP served as the control (A). (B) Cotransfection of EGFP-Rab5 ${ }^{\text {WT }}$ with APP-mCherry. Cotransfection of mCherry-Rab5 ${ }^{\text {WT }}$ with (C) C99-GFP or (D) C83-GFP. Insets: Zoom-in ( $\left.\times 2.5\right)$ images of the selected areas. Scale bars: $10 \mu \mathrm{m}$.

C99-GFP each was expressed in PC12M cells. EGFP was used as a negative control and Rabex-5 as a positive control. Twenty-four hours after transfection, GTP pull-down assays were used to measure the level of activated Rab5 (56). Each of the APP constructs and C99 induced a large increase in activated Rab5, as did Rabex-5 (Figure 5, A and B). In contrast, neither C83-GFP nor AICD-GFP induced increased Rab5 activation (Figure 5, A and B; see also Supplemental Figure 2A). The data thus demonstrate that full-length $\mathrm{APP}, \mathrm{APP}^{\mathrm{SWE}}, \mathrm{APP}^{\mathrm{M} 596 \mathrm{~V}}$, and C99 all induced activation of Rab5 and enlargement of early endosomes.

To ask whether increased levels of $\mathrm{C} 99$, not $\mathrm{A} \beta$ peptides, were responsible for the increase in Rab5 endosomal size, we tested a GSI (BMS-299897) that blocks production of all A $\beta$ species while increasing the level of C99 (40, 63-70). Treatment of PC12 cells with $1 \mu \mathrm{M}$ GSI for 48 hours caused a marked increase in the level of CTFs, as detected by immunoblotting with an antibody specific to the C-terminus of APP, with little effect on the level of full-length APP (Figure 5C). In comparison with vehicle treatment, GSI treatment of cells expressing mCherry-Rab5 ${ }^{\mathrm{WT}}$ for 48 hours induced apparent enlargement of mCherry-Rab5 ${ }^{\mathrm{WT}}$ endosomes (Figure 5D). Quantitative analysis showed that GSI treatment induced a significant decrease in the size of smaller mCherry-Rab5 ${ }^{\mathrm{WT}}$ puncta $\left(0.2-0.4 \mu \mathrm{m}^{2}\right)$, with a concomitant increase in larger puncta $\left(0.6\right.$ to $\left.\geq 1.0 \mu \mathrm{m}^{2}\right)$ (Figure $\left.5 \mathrm{E}\right)$. 

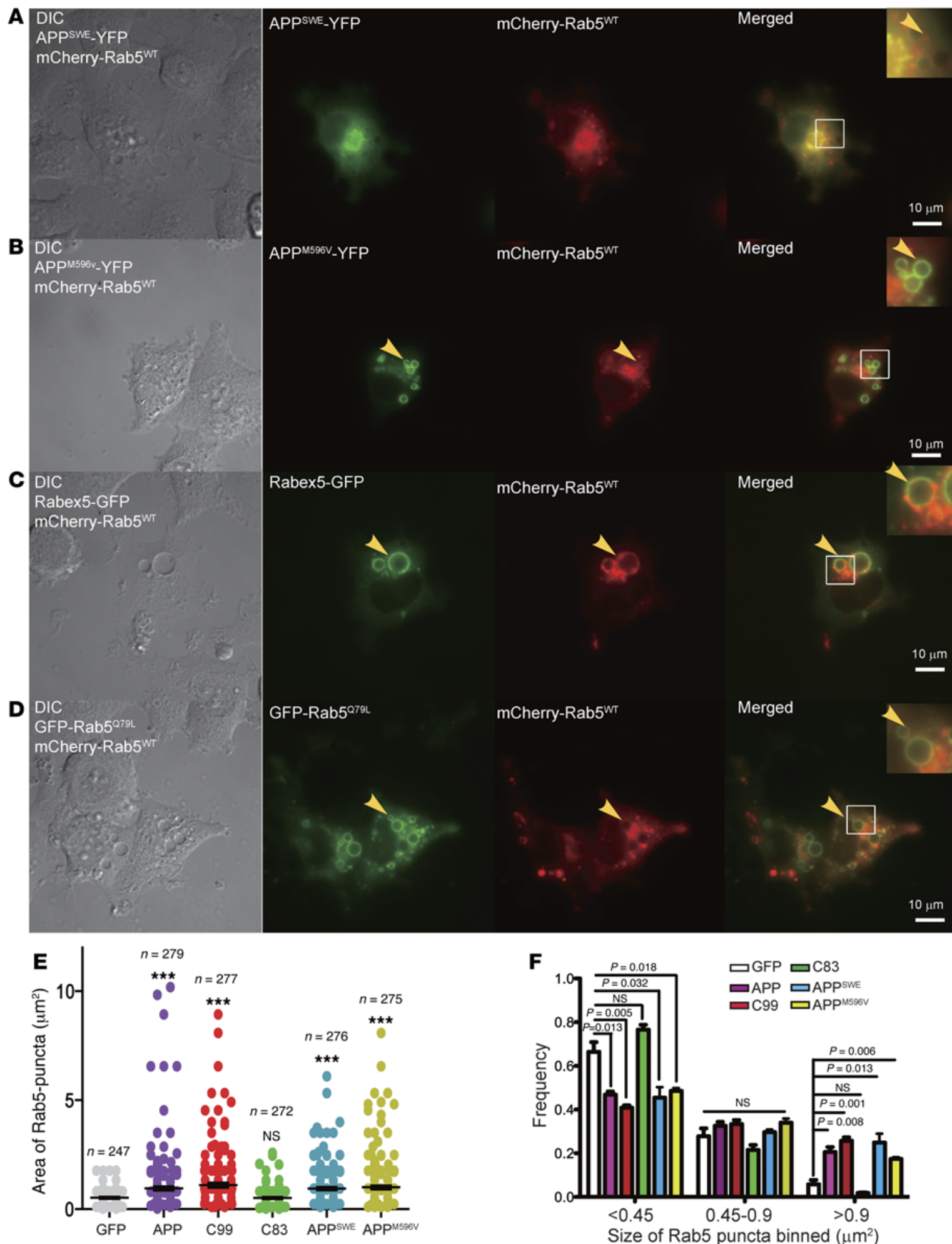

Figure 4. APP ${ }^{\mathrm{SWE}}$ and APP ${ }^{\mathrm{M} 96 \mathrm{~V}}$ induced the enlargement of Rab5 ${ }^{+}$endosomes in PC12M cells. As in Figure 3, mCherry-Rab5 ${ }^{\mathrm{WT}}$ was cotransfected into PC12M cells with APP ${ }^{\text {SWE }}$-YFP (A), APP ${ }^{M 966}-$ YFP (B), Rabex-5-GFP (C), or GFP-Rab5 ${ }^{\text {Q79L }}$ (D). Scale bars: $10 \mu \mathrm{m}$. Rab5 ${ }^{+}$endosomes are indicated by arrowheads. Insets: Zoom-in ( $\times 2.5)$ images of the selected areas. The size of Rab5+ endosomes for each APP constructs was quantified using ImageJ, and the results are shown in $\mathbf{E}\left({ }^{* *} P<0.001\right)$, with the size distribution of Rab5+ endosomes shown in $\mathbf{F}$. All $P$ values were calculated using Student's $t$ test. 

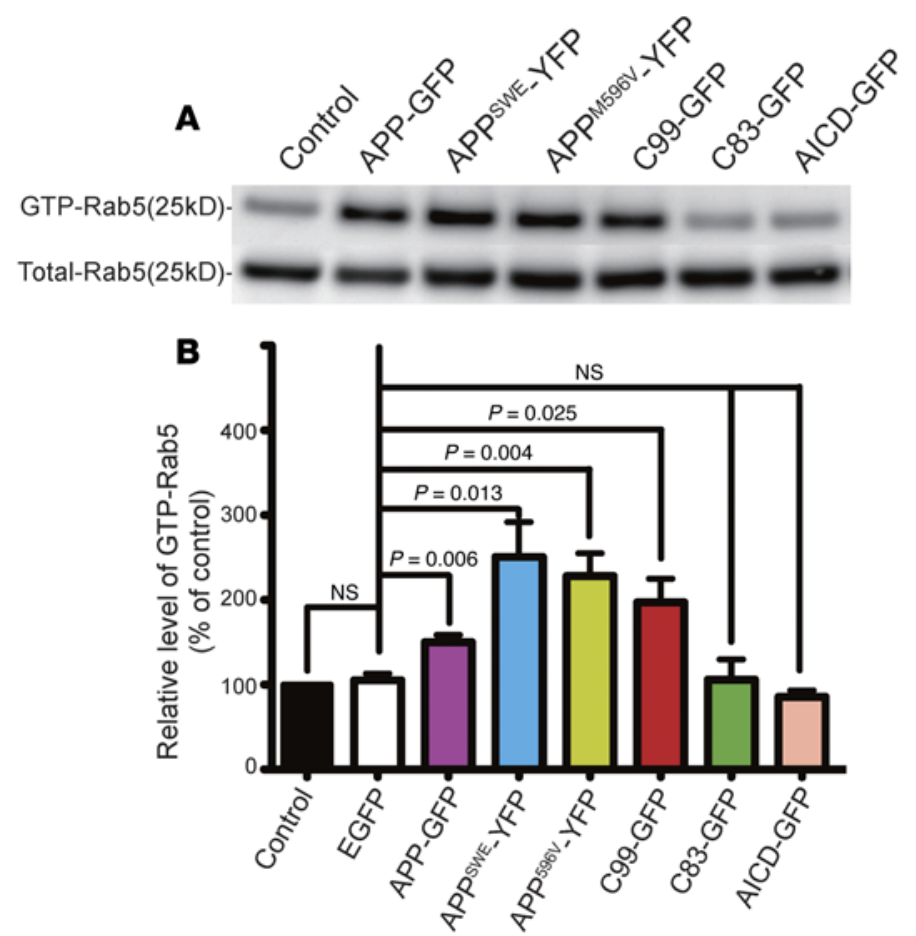

C

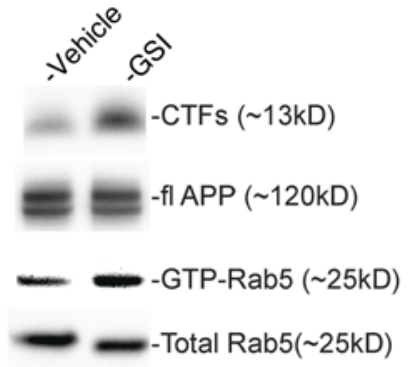

D
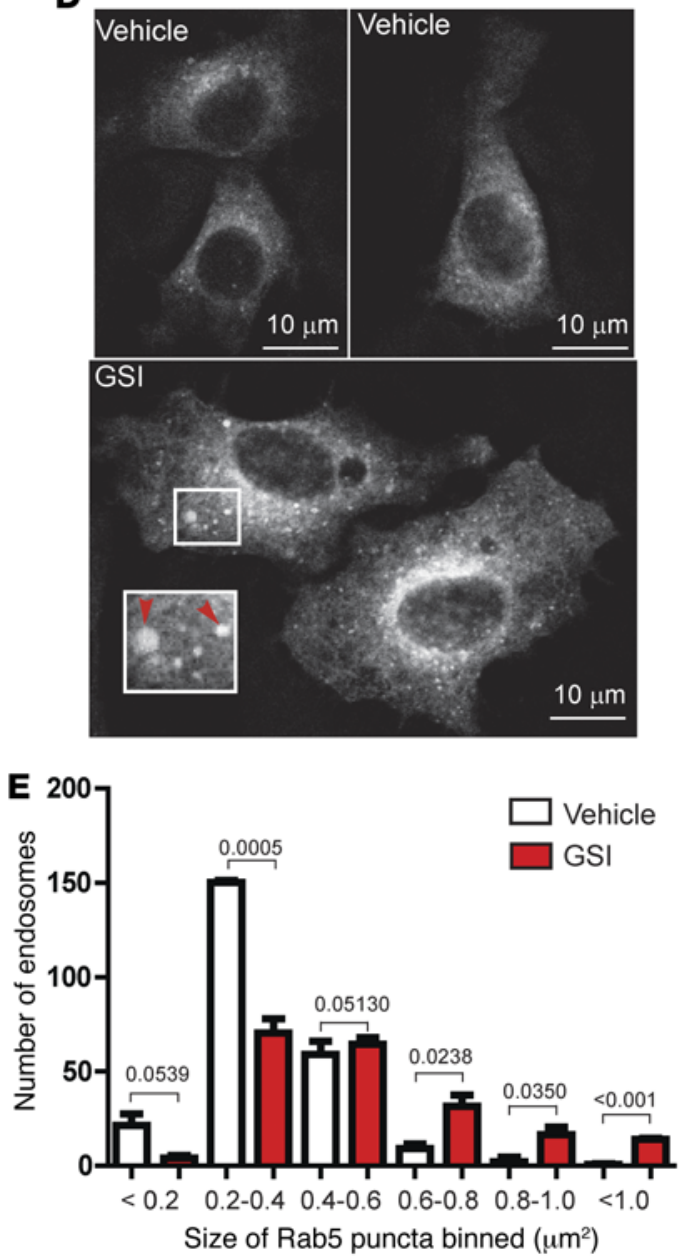

Figure 5. APP, APP mutants, or $\mathbf{C 9}$ induced hyperactivation of Rab5 in PC12M cells. PC12M cells were cultured and transfected with the indicated constructs. The levels of GTP-Rab5 were assayed as in Figure 2. The levels of total Rab5 were blotted as loading controls (A). The results were quantitated and are presented in B. The level of GTP-Rab5 in untransfected cells was set at 100\%. (C) PC12M cells were treated with either vehicle or $1 \mu$ M GSI for 24 hours, and cell lysates were analyzed by SDS-PACE/immunoblotting with the indicated antibodies. Both full-length APP (fI APP) and APP CTFs are shown. The levels of activated Rab5 and total Rab5 are also shown. (D) PC12M cells were transfected with mCherry-Rab5WT for 24 hours and were treated with either $1 \mu \mathrm{M} \mathrm{CSI}$ or vehicle for another 48 hours. Cells were fixed and analyzed by use of a Leica TCS SPE confocal microscope with a $\times 63$ oil objective lens. Representative images of mCherry-Rab5 ${ }^{\mathrm{WT}}$ puncta are shown. Enlarged mCherry-Rab5 ${ }^{\mathrm{WT}}$ puncta by GSI treatment are highlighted in the inset ( $\times 1.6$; arrowheads). Scale bars: $10 \mu \mathrm{m}$. The sizes of mCherry-Rab5 ${ }^{\mathrm{WT}}$ puncta from 25-30 transfected cells were quantitated, and the size distribution pattern is shown in $\mathbf{E}$. Data represent mean \pm SEM of at least 3 independent experiments. All corresponding $P$ values were calculated using Student's $t$ test.

To test whether increased Rab5 activation was necessary for the effect of APP-GFP or C99-GFP on endosomal size, we coexpressed these constructs with a dominant-negative mutant, mCherry-Rab5 $5^{\mathrm{S} 34 \mathrm{~N}}(22,55)$, which resulted in the disappearance of bright intracellular GFP foci (Supplemental Figure 1, D versus B). Quantitative analyses revealed a reduction in both the size (Supplemental Figure 1E) and number (Supplemental Figure 1F) of GFP puncta, as was evident in the frequency distribution of $\mathrm{GFP}^{+}$ vesicles (Supplemental Figure 1G). We thus conclude that overexpression of APP or C99 acted through Rab5 activation to increase size across the entire population of early endosomes.

Full-length APP or C99 inhibited NGF-mediated neurite outgrowth in PC12M cells. Endocytosis is essential for NGF-mediated neurite outgrowth in PC12 cells $(71,72)$. Increased Rab5 activation compromised endocytic trafficking of NGF signaling complexes and blocked NGF signaling in PC12 cells (71). PC12M cells were transfected with EGFP, full-length APP-GFP, C99-GFP, C83-GFP, APP ${ }^{\mathrm{M} 596 \mathrm{~V}}-\mathrm{YFP}$, or Rabex-5-GFP. NGF (50 ng/ml) was added to the culture 24 hours after transfection to induce differentiation for 2 days. The length and number of neurites were quantitated. Representative images are shown in Figure 6A, and the quantitative results in Figure 6, B and C. Cells transfected with EGFP demonstrated numerous long, neuron-like processes, some terminating in growth cone-like structures; on average there were $3.75 \pm 0.17$ neurites/cell (Figure 6B). The mean length of neurites in EGFPexpressing cells was $91.98 \pm 4.08 \mu \mathrm{m}$ (Figure $6 \mathrm{C}$ ). PC12M cells transfected with APP-GFP, APP ${ }^{\mathrm{M} 96 \mathrm{~V}}$-YFP, or C99-GFP demonstrated a significant reduction in both the number of neurites/cell 
A

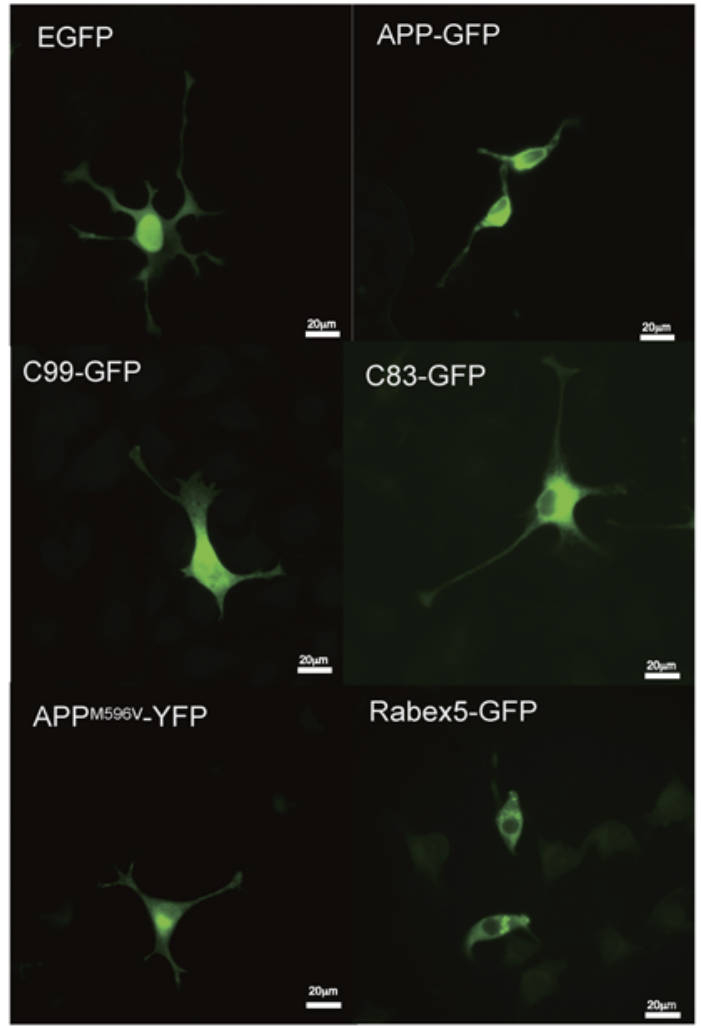

D

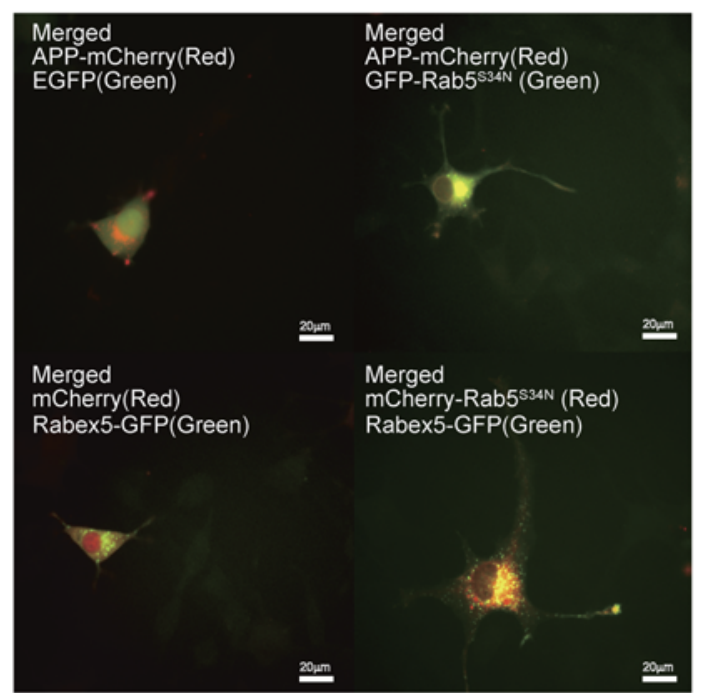

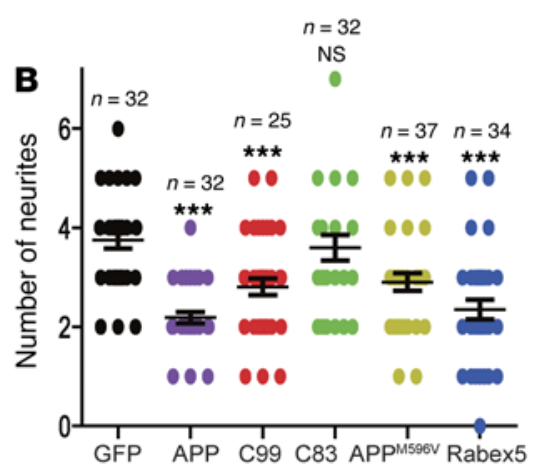
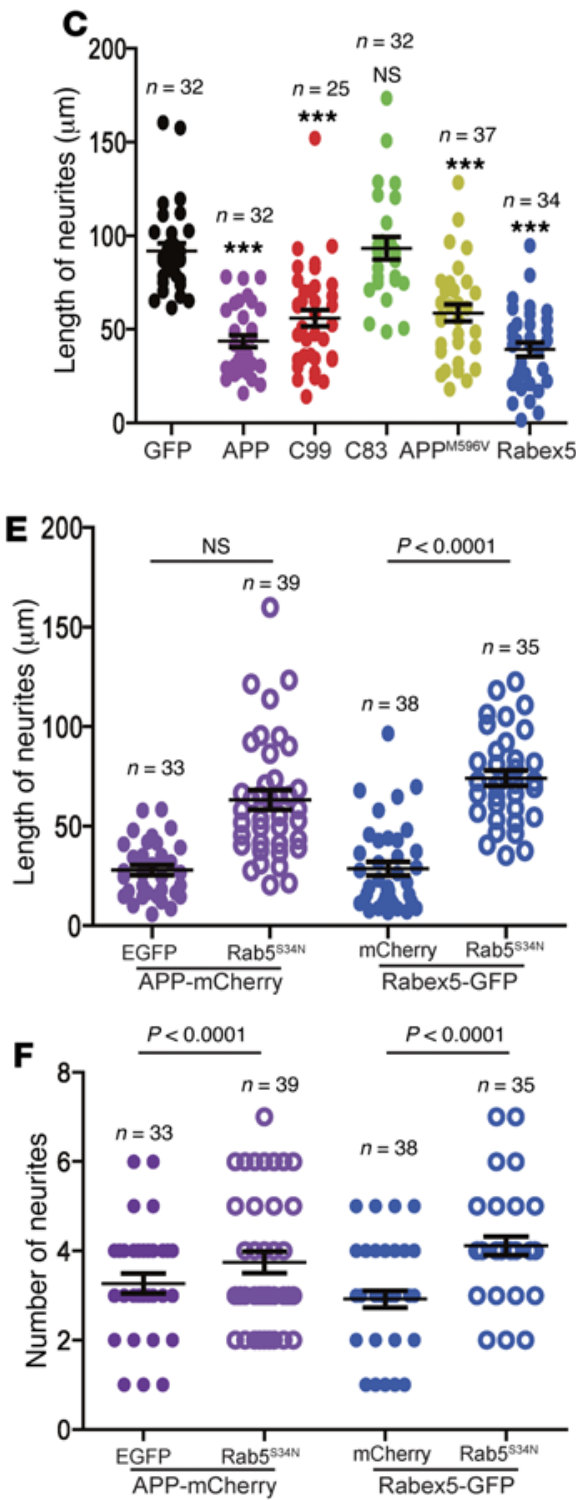

Figure 6. APP, APP mutants, or C99 inhibited NGF-

induced neurite out-

growth that was rescued by Rab5 ${ }^{\text {S34N. }}$. PC12M cells were transfected with the indicated plasmids and treated with $50 \mathrm{ng} / \mathrm{ml}$ NGF for 48 hours. The length and the number of neurites of each cell were measured and quantitated using Image). Representative images are shown in $\mathbf{A}$. The number of the neurites/cell (B) and the average length (C) in each condition are measured, and the results are shown. (D-F) Rab5 $5^{534 \mathrm{~N}}$-GFP or Rab $5^{534 \mathrm{~N}}$-mCherry was cotransfected with APPmCherry or Rabex-5-GFP into PC12M cells (D). Cells were then induced for differentiation. The number of the neurites/cell (E) and the average length $(\mathbf{F})$ of each condition were measured, and the results are shown. All $P$ values were calculated using Student's $t$ test; ${ }^{* *} P<$ 0.001 . Scale bars: $20 \mu \mathrm{m}$.

$(2.19 \pm 0.11,2.91 \pm 0.18$, and $2.81 \pm 0.17$, respectively) and the mean length of neurites $(43.73 \pm 3.23 \mu \mathrm{m}, 58.8 \pm 4.62 \mu \mathrm{m}$, and $55.95 \pm$ $4.45 \mu \mathrm{m}$, respectively) (Figure 6, B and C). In contrast, expression of C83-GFP resulted in no significant differences relative to the EGFP control in these measures (neurite number/cell: $3.60 \pm$ 0.26; mean neurite length: $93.28 \pm 6.09 \mu \mathrm{m}$ ) (Figure 6, A-C). To test whether increased Rab5 activation would result in reduction in neurite outgrowth and number, we tested Rabex-5-GFP, which induced hyperactivation of Rab5 (Figure 4C and Supplemental Figure 3). Neurites were significantly fewer in number $(2.35 \pm$ 0.20 neurites/cell) and shorter $(39.33 \pm 3.66 \mu \mathrm{m})$ with expression of Rab5ex-5-GFP (Figure 6, A-C).

To determine whether the decrease in neurite elaboration and growth detected in studies involving overexpression of APP con- 


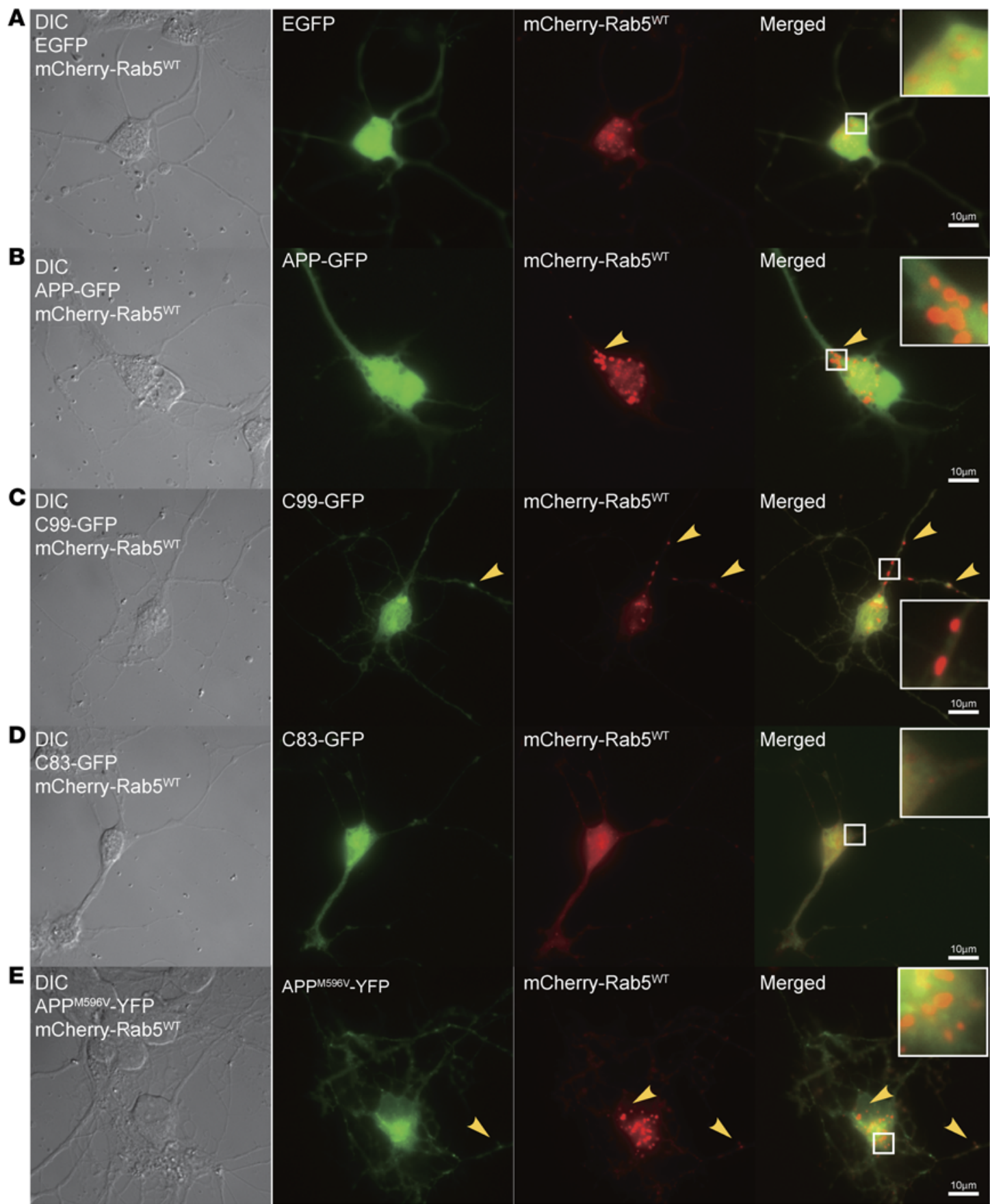

Figure 7. APP, APPM596v, or C99 induced the enlargement of Rab5+ endosomes in BFCNs. Primary rat E18 BFCNs were cultured on poly-L-lysine-coated glass coverslips. Neurons (DIV6) were transfected with the indicated plasmids for 24-48 hours. Live imaging was performed as described in Methods. Images of DIC, red, and green channels were captured. Representative images of BFCNs are shown for cotransfection: mCherry-Rab5 ${ }^{\mathrm{WT}} / \mathrm{ECFP}$ (A), mCherry-Rab5 ${ }^{\mathrm{WT}}$ / APP-GFP (B), mCherry-Rab5 ${ }^{W T}$ /C99-GFP (C), mCherry-Rab5WT/C83-GFP (D), mCherry-Rab5 ${ }^{\text {WT } / A P P M 596 v-Y F P ~(E) . ~ A r r o w h e a d s ~ i n d i c a t e ~ R a b 5 ~}{ }^{+}$endosomes. Over-

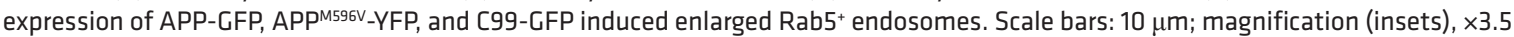

structs were due to increased Rab5 activation, we cotransfected GFP-Rab5 $5^{\mathrm{S} 34 \mathrm{~N}} / \mathrm{APP}-\mathrm{mCherry}$ or mCherry-Rab5 $5^{\mathrm{S} 34 \mathrm{~N}} / \mathrm{Rabex}^{-5-G F P}$ into PC12M cells. Cells were then treated with NGF $(50 \mathrm{ng} / \mathrm{ml})$ to induce neurite outgrowth. Cells coexpressing Rab5 $5^{\mathrm{s} 34 \mathrm{~N}} / \mathrm{APP}$ or Rab5 $5^{\mathrm{S} 34 \mathrm{~N}} /$ Rabex -5 showed a statistically significant increase in neurite length $(63.21 \pm 4.88 \mu \mathrm{m}$ and $74.14 \pm 3.84 \mu \mathrm{m}$, respectively) compared with cells expressing APP or Rabex-5 alone (Figure 6, $\mathrm{D}$ and $\mathrm{E})$. Expression of Rab5 $5^{\mathrm{S} 34 \mathrm{~N}}$ also increased the number of neurites per cell; for those also expressing Rabex-5, the increase was statistically significant (Figure 6F). We thus conclude that the disruption of the effects of NGF signaling on neuronal differentiation mediated by increased expression of APP and C99 was due, at least in part, to increased activation of Rab5.

Because sustained activation of the MAPK pathway contributes significantly to neuronal differentiation of PC12 cells in response to NGF, we assessed activation of Erk1/2 in cells that 


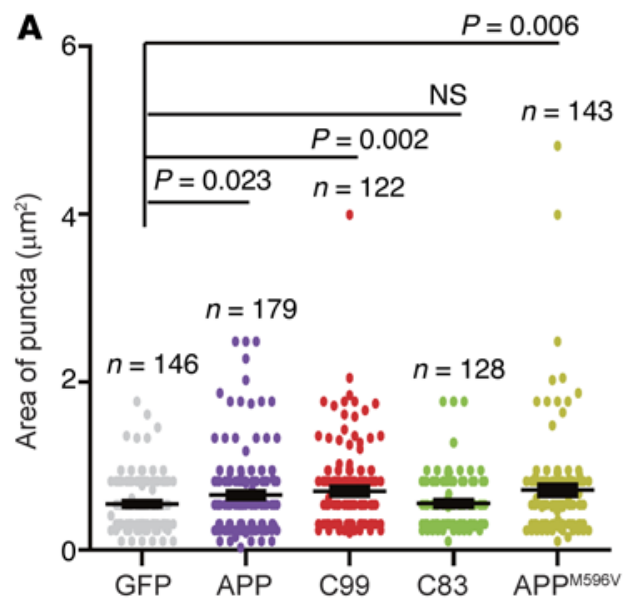

B

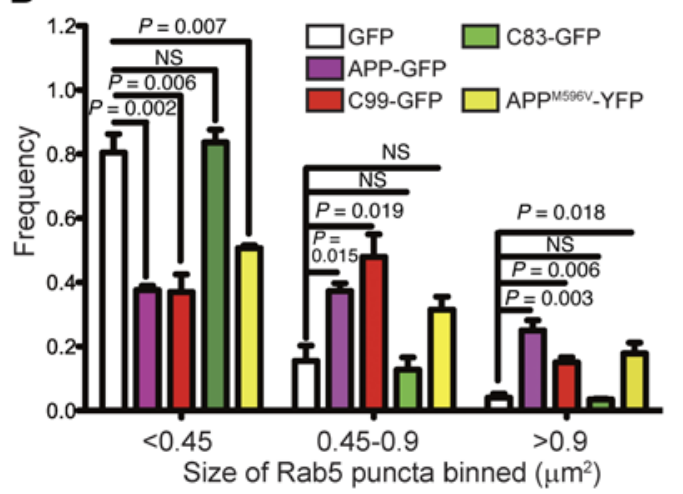

C

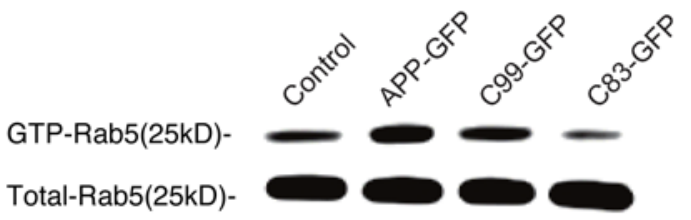

D

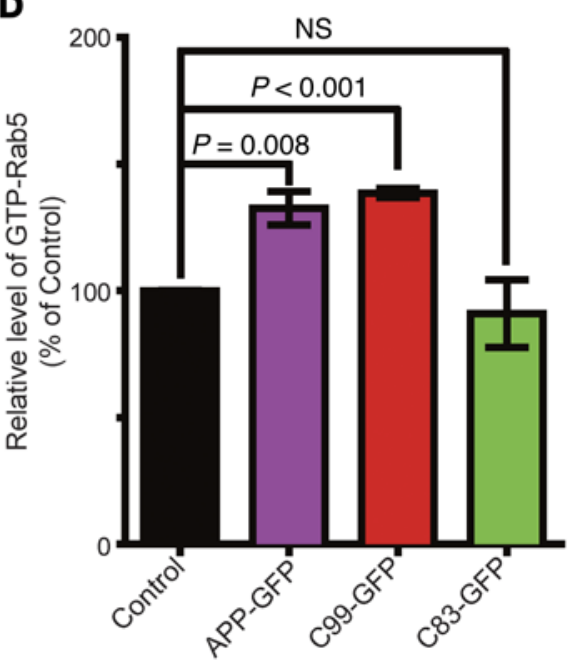

Figure 8. APP and C99 increased the level of GTP-Rab5 in BFCNs.

(A) The size of Rab5+ endosomes in primary BFCNs from experiments in Figure 7 was quantified using Image). The endosomal size distribution for APP-GFP, APPM596V_YFP, and C99-GFP showed a shift in sizes from smaller to larger binned areas as compared with that for EGFP and C83-GFP (B). Data represent the mean value of three independent experiments. ( $C$ and $\mathbf{D}$ ) The levels of GTP-Rab5 and total Rab5 were also measured in BFCNs that expressed these various constructs. The level of GTP-Rab5 in untransfected cells was set at $100 \%$ (D). Data represent mean \pm SEM of at least 3 independent experiments. All $P$ values were calculated using Student's $t$ test. expressed C99 and C83 following NGF treatment. Consistent with earlier studies (73-75), expression of C99 markedly suppressed NGF-induced activation of Erk1/2 at both 5 and 30 minutes (Supplemental Figure 2, B and C). While having no effect at 5 minutes, C83 induced a small decrease in pErk1/2 at 30 minutes. Therefore, APP and C99, and to lesser extent C83, impacted NGF signaling through the MAPK pathway.

Full-length APP or C99 induced enlargement of earlyendosomes and atrophy of BFCNs. BFCNs are selectively impacted in AD and DS (50, $51,76-82)$. NGF plays a critical role in supporting the differentiation and maintenance of these neurons $(27,48,79,83-87)$. The Ts65Dn mouse model recapitulates age-related atrophy of BFCNs, to which $A P P$ gene dose contributes prominently $(28,38)$. Importantly, neuronal atrophy is correlated with $A P P$ gene dose-mediated disruption of retrograde NGF transport to BFCNs from the hippocampus (28). We asked whether APP or C99 compromised NGF signaling and trafficking in BFCNs in vitro. Following cotransfection of primary rat E18 BFCNs with mCherry-Rab5 ${ }^{\mathrm{WT}}$ and APP-GFP or various CTFGFP constructs at 6 days in vitro (DIV6), we used live cell imaging to examine endosomes. Representative images are shown in Figure 7, A-E. As in PC12M cells, BFCNs overexpressing APP-GFP (Figure 7B, inset), C99-GFP (Figure 7C, inset), or APP ${ }^{\mathrm{M} 596 \mathrm{~V}-Y F P}$ (Figure 7E, inset) exhibited enlarged Rab5 ${ }^{+}$endosomes, all showing extensive colocalization with Rab5. EGFP or C83-GFP did not cause these changes (Figure 7, A and D).

We quantitated the size of Rab5 $5^{+}$puncta in BFCNs that overexpressed EGFP, APP-GFP, C99-GFP, C83-GFP, or APP ${ }^{\mathrm{M} 596 \mathrm{~V}-Y F P}$
(Figure 8A). The results showed an increase in size of $18 \%$ for APPGFP, 27\% for C99-GFP, and 29\% for APP ${ }^{\mathrm{M} 596 \mathrm{~V}-\mathrm{YFP}}$ in comparison to BFCNs expressing EGFP (Figure 8A). The distribution of Rab5 ${ }^{+}$ puncta in BFCNs expressing these constructs also showed a shift in sizes from smaller to larger binned areas (Figure 8B), reflecting an apparent effect on the entire population of Rab5 $5^{+}$endosomes. We measured the level of GTP-bound Rab5. The results showed increases in activated Rab5, in comparison to the control, of $45.7 \%$ and $52.2 \%$ in BFCNs transfected with APP-GFP and C99-GFP, respectively $(P<0.05)$ (Figure $8, C$ and $D)$. In contrast, overexpression of C83-GFP did not increase the level of GTP-Rab5 (Figure 8, C and D); nor did C83 expression induce an increase in the size of Rab5 $5^{+}$endosomes. Therefore, increased expression of APP or C99, but not C83, induced activation of Rab5 and enlargement of $\mathrm{Rab5}^{+}$early endosomes in BFCNs.

C99, not C83, disrupted retrograde transport of NGF and inhibited NGF signaling in BFCNs. Retrograde axonal transport and signaling of NGF support the maintenance and function of BFCNs $(46,47,51,88)$. Because increased App gene dose reduced retrograde axonal transport of NGF from hippocampus to the basal forebrain in Ts65Dn mice (28), we asked whether APP and C99 overexpression impacted retrograde transport of NGF in BFCNs. Quantum dot-labeled NGF (QD-NGF) was used to track the axonal transport of NGF by live cell imaging $(89,90)$. Primary cultures of rat E18 BFCNs were maintained in microfluidic culture chambers, in which axons are separated from the corresponding cell bodies (91). To examine the Rab5 ${ }^{+}$endosomes in axons, we 
A

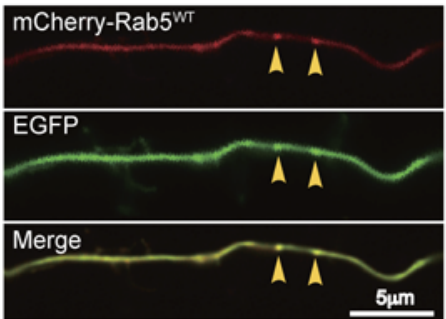

D
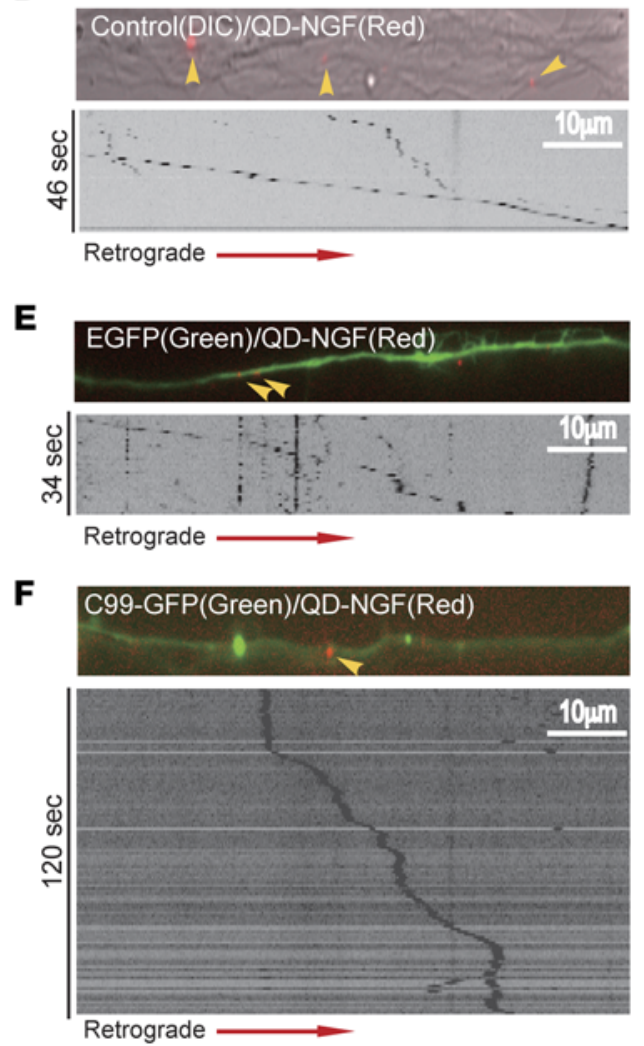

G

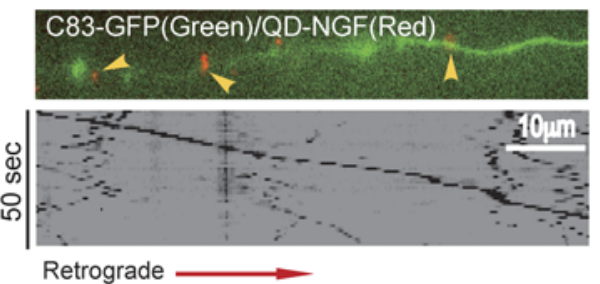

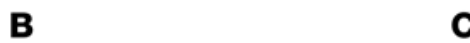
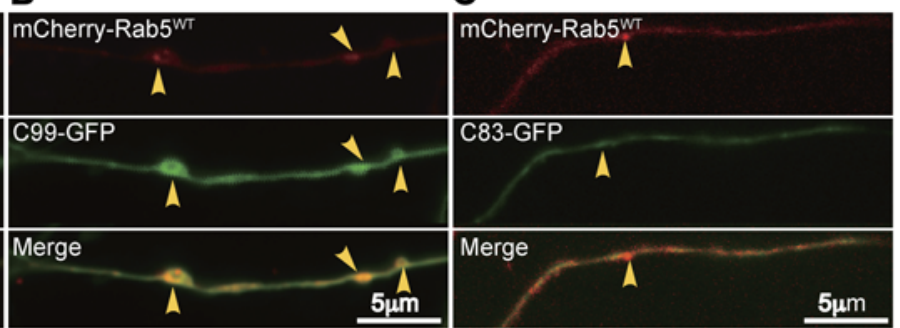

H

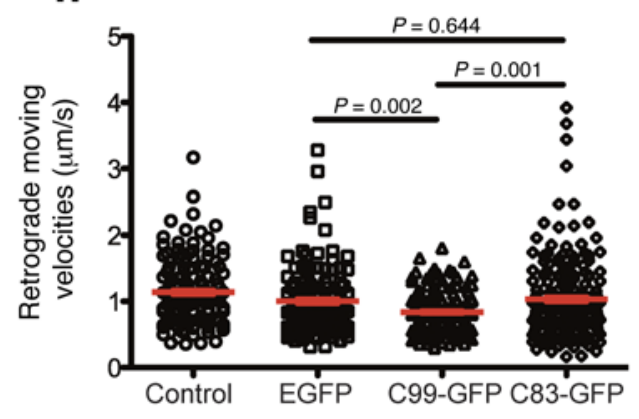

I
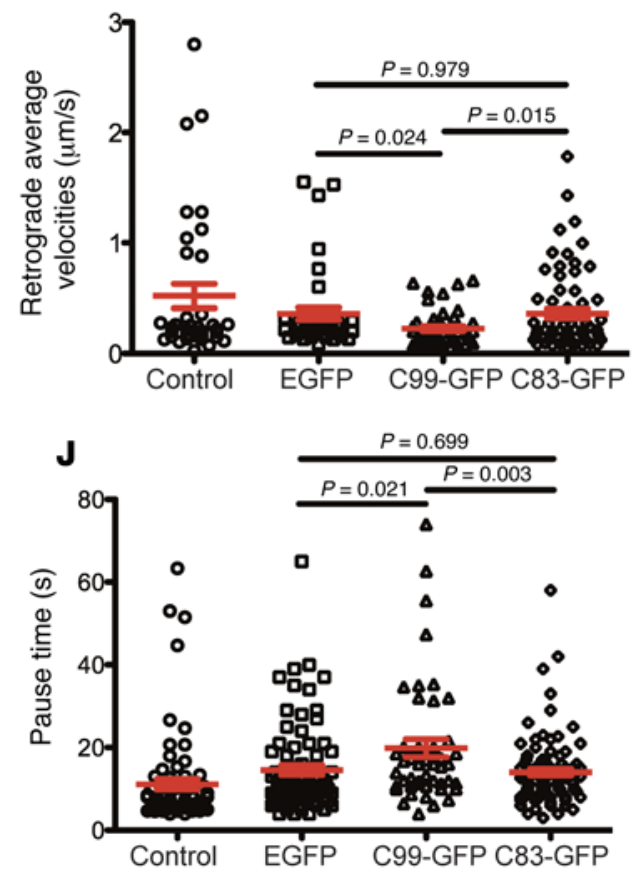

Figure 9. C99 inhibited retrograde axonal transport of NGF in BFCNs. (A) Rat E18 BFCNs were cultured in microfluidic chambers and were cotransfected with the indicated expression vectors. Live imaging was performed as described in Methods. Representative images of axons of BFCNs are shown for mCherry-Rab5 ${ }^{\mathrm{WT}} /$ EGFP (A), mCherry-Rab5/C99-GFP (B), and mCherry-Rab5/C83-GFP (C) Arrowheads indicate the enlarged $\mathrm{Rab5}^{+}$structures that colocalized with C99-GFP. Scale bars: $5 \mu \mathrm{m}$. (D-G) Representative images and kymographs of axonal transport of QD-NGF signals. Scale bars: $10 \mu \mathrm{m}$. Retrograde movement $(\mathbf{H})$, average velocities (I), and pause time (J) of the QD-NGF signals in axons were quantitated. Significantly slowed retrograde transport and longer pause time are seen for C99-GFP-transfected axons as compared with untransfected control or with C83-GFP-transfected axons (C-E). Data represent mean \pm SEM of at least 3 independent experiments. All $P$ values were calculated using 1-way ANOVA. transfected BFCNs at DIV7 with mCherry-Rab5 ${ }^{\mathrm{WT}}$ together with EGFP, C99-GFP, or C83-GFP (Figure 9, A-C). The axons of neurons cotransfected with EGFP showed diffuse mCherry-Rab5 ${ }^{\text {WT }}$ signals with occasional distinct rounded puncta (Figure 9A, arrowheads). A similar appearance was found in axons of neurons cotransfected with C83-GFP and mCherry-Rab5 ${ }^{\mathrm{WT}}$, with colocalization of the latter in small puncta (Figure 9C). In contrast, the axons of C99-GFP-transfected neurons showed distinct differences. The signals for mCherry-Rab5 ${ }^{\mathrm{WT}}$ were concentrated within varicosities containing enlarged $\mathrm{Rab5}^{+}$structures; puncta were also seen between varicosities, with most puncta showing colocalization of Rab5 and C99-GFP (Figure 9B, arrowheads).
To ask whether overexpression of C99 impacted axonal transport, we examined live imaging of axonal transport of QD-NGF 24 hours after transfection. Time-lapse image series were captured, and kymographs were generated; representative images with corresponding kymographs for each condition are shown (Figure 9, D-G, and Supplemental Videos 1-4). When compared with either EGFP- or C83-GFP-transfected neurons, there was a significant reduction in both the moving (i.e., instantaneous) (Figure 9H) and average (Figure 9I) velocities of QD-NGF in axons of BFCNs expressing C99-GFP. The moving velocity of QD-NGF in C99-GFP-transfected neurons was significantly reduced with respect to both EGFP-expressing neurons (by approximately $16.5 \% ; P=0.002$ ) and C83-GFP-expressing 
A
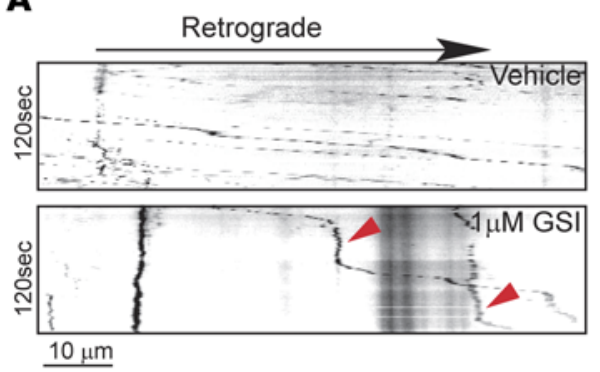

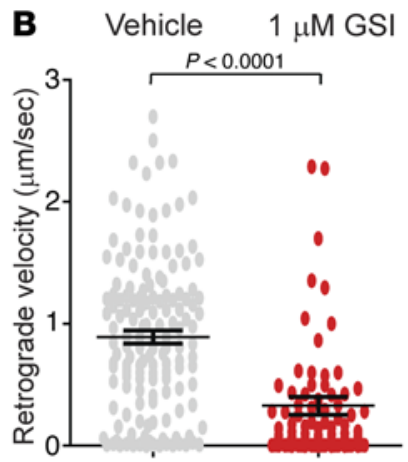

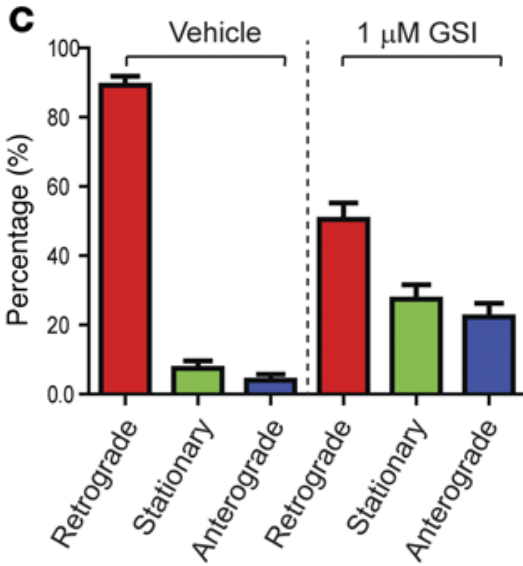

Figure 10. GSI induced hyperactivation of Rab5 and inhibited retrograde axonal transport of NGF in BFCNs. Rat E18 BFCNs (DIV6) were cultured in microfluidic chambers, and both the cell body and the distal axonal chamber were treated with either vehicle or $1 \mu \mathrm{M}$ GSI for 2 hours. Live imaging of QD-NGF was performed as described in Methods. (A) Representative kymographs derived from time-lapse image series of axonal transport of QD-NGF from vehicle- and GSI-treated samples. (B) Retrograde moving speed and (C) breakdowns of transport directionalities (retrograde, stationary, and anterograde) for vehicle- and CSI-treated samples. Data represent mean \pm SEM of at least 3 independent experiments. The $P$ value was calculated using Student's $t$ test.

neurons (by 13.5\%; $P=0.001$ ) (Figure 9H). The moving velocities for QD-NGF did not differ among control, EGFP-expresssing, and C83-GFP-expressing neurons $(P=0.644)$ (Figure $9 \mathrm{H})$. The average velocities of $\mathrm{QD}-\mathrm{NGF}$ in $\mathrm{C} 99$-GFP-transfected neurons were also reduced, to $62.9 \%(P=0.024)$ of those in EGFP neurons and to $62.6 \%$ $(P=0.015)$ of those in C83-GFP neurons (Figure 9I). Again, the average velocities of QD-NGF did not differ among control, EGFPexpressing, and C83-GFP-expressing neurons ( $P=0.979$ ) (Figure 9I). In addition, we examined the number of stationary (pause) periods between moving segments. Such periods were significantly increased for QD-NGF signals within axons of C99-GFP neurons. The increase amounted to $36.4 \%(P=0.021)$ as compared with EGFP- and $41.7 \%$ $(P=0.003)$ as compared with C83-GFP-transfected neurons, respectively (Figure 9J). C99-GFP thus induced significant changes in retrograde axonal transport of NGF in BFCNs.

GSIs that block production of all $A \beta$ species while increasing the level of CTFs, including C99 (63-70), caused a marked increase in Rab5 activation and enlargement of Rab5 $5^{+}$endosomes (Figure 5, C-E). We reasoned that GSI would disrupt retrograde axonal transport of NGF. Using the same culture system as for BFCNs overexpressing APP and its products, we found that a 24-hour pretreatment with $1 \mu \mathrm{M}$ GSI resulted in significant impairment of retrograde axonal transport of NGF. Representative kymographs (vehicle versus GSI) showed significant differences from control cultures (Figure 10A and Supplemental Videos 5 and 6). GSI treatment caused a marked decrease in moving velocity (Figure 10B); moreover, it reduced the percentage of NGF moving retrogradely (Figure 10C) while increasing the percentage that moved anterogradely or paused (Figure 10C). We conclude that C99 disrupts retrograde axonal transport of NGF in BFCNs.

Atrophy of BFCNs induced by C99 was prevented by dominantnegative Rab5. Reduced axonal transport of NGF would result in decreased transport of trophic signals from axons to cell bodies. To measure NGF signaling in BFCNs, we examined immunostaining for the phosphorylated form of cAMP response element-binding protein (CREB), an important element downstream of axonally transported NGF-TrkA signals (46, 47, 92). E18 BFCN neurons that were cultured in microfluidic chambers were transfected with EGFP (Supplemental Figure 4), C99-GFP (Supplemental Figure 3), or C83-GFP (Supplemental Figure 4), followed by immunostaining of cell bodies with a specific antibody to pCREB. NGF $(50 \mathrm{ng} / \mathrm{ml})$ was supplied to only the axonal chambers for 72 hours before examination of pCREB. In comparison to EGFP- or C83-GFP-expressing neurons, there was a marked reduction in PCREB signals in C99GFP-expressing neurons (Supplemental Figure 4).

Since the size of BFCN soma is regulated by NGF (93), we examined the effect APP overexpression on NGF signaling by measuring the soma size of BFCNs 48 hours after transfection with EGFP, APP, C99, or C83; in each case, the media bathing cells contained $50 \mathrm{ng} / \mathrm{ml} \mathrm{NGF}$. As shown in Figure 11A, the area of BFCN cell bodies that overexpressed APP-GFP $(P=0.0075)$ and C99-GFP $(P=0.0488)$ was markedly decreased. The sizes of BFCNs that overexpressed C83-GFP tended to be smaller than those transfected with EGFP, but the effect failed to reach significance $(P=$ 0.3144) (Figure 11A). To ask whether C99-induced atrophy could be overcome by increasing the concentration of NGF, we set up microfluidic chamber cultures and transfected cells with either EGFP or C99-GFP. The cultures were maintained in 10, 50, and $100 \mathrm{ng} / \mathrm{ml}$ NGF provided only in the axonal chamber for 72 hours before measurement of the size of neuronal somata. For EGFP-expressing neurons, increasing NGF concentration from 10 to $50 \mathrm{ng} / \mathrm{ml}$ resulted in a marked increase in size of approximately 150 to 190 $\mu^{2}{ }^{2}$ (Figure 11B). In contrast, increasing NGF from 10 to $50 \mathrm{ng} / \mathrm{ml}$ had no appreciable effect on soma size in C99-GFP-expressing neurons (Figure 11B). At 100 ng/ml NGF, C99-GFP-expressing neurons showed a small increase in soma size, but the increase was not significant, and cell body size was still smaller than that of EGFP-expressing neurons (Figure 11B). Therefore, C99 overexpression markedly impacts retrograde axonal trafficking of NGF signaling as well as the soma size of BFCNs.

To determine whether activated Rab5 contributed to reduced NGF retrograde transport and signaling in BFCNs, 
A

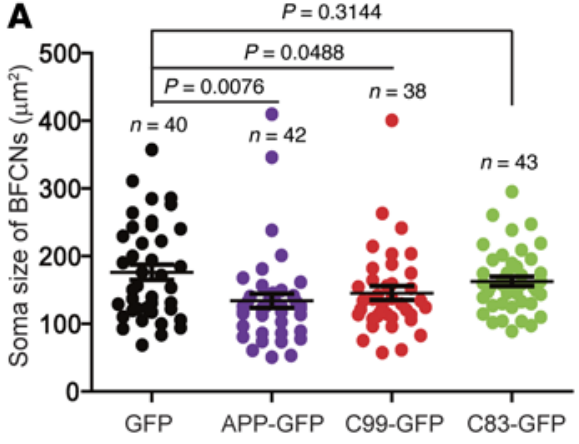

B

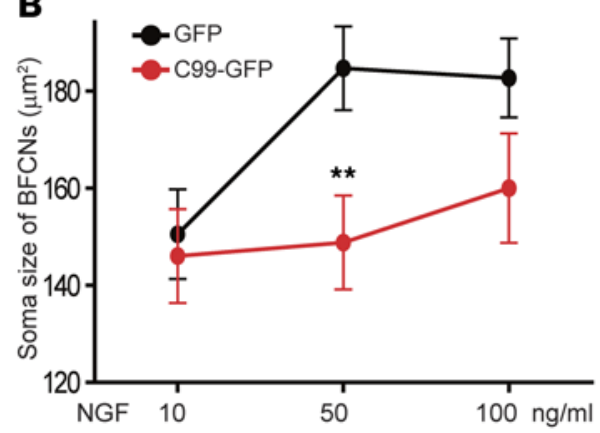

C

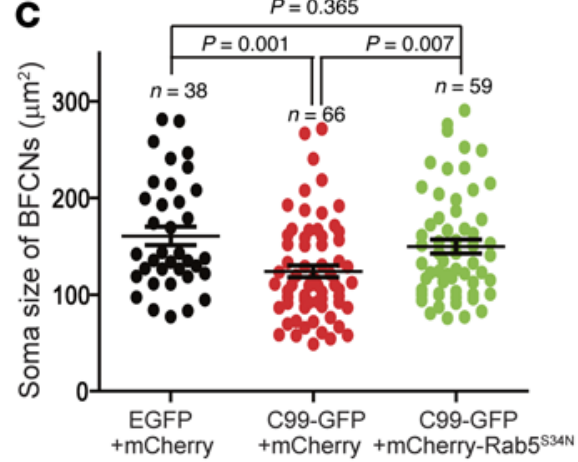

Figure 11. C99 induced-atrophy of BFCNs was rescued by Rab5534N. Rat E18 BFCNs were cultured in microfluidic chambers as described in Methods. (A) Neurons were transfected with the indicated plasmids for 48 hours, and the soma profiles of BFCNs were measured and quantified using Image). (B) BFCNs were transfected with EGFP or C99 and were maintained in medium containing 10,50 , or $100 \mathrm{ng} / \mathrm{ml} \mathrm{NGF}$ for 48 hours; soma sizes of BFCNs were measured as in A. (C) BFCNs were transfected with the indicated plasmids for 48 hours; the soma profiles of BFCNs were measured as in $\mathbf{A}$ and $\mathbf{B}$. All $P$ values were calculated using Student's $t$ test.

we cotransfected C99-GFP with the dominant-negative Rab5 mutant (mCherry-Rab5 ${ }^{\mathrm{S} 34 \mathrm{~N}}$ ) at DIV7 into rat BFCNs cultured in microfluidic chambers. The cultures were maintained for another 72 hours with only axonally added $50 \mathrm{ng} / \mathrm{ml} \mathrm{NGF}$. The mean soma size of these neurons was markedly increased as compared with control neurons expressing C99-GFP and mCherry $(P=0.007)$ (Figure $11 C)$. The size of BFCNs that coexpressed mCherry-Rab5 $5^{\mathrm{S} 34 \mathrm{~N}}$ was equivalent to that of BFCNs expressing EGFP and mCherry $(P=0.365)$ (Figure $11 C)$. Therefore, $\mathrm{C} 99$ acts to induce Rab5 activation to disrupt retrograde NGF signaling, resulting in atrophy of BFCNs. Taken together, the results indicate that APP and C99 both act through Rab5 activation to impact the structure and function of early endosomes, causing deleterious effects on the trophic status of BFCNs.

To validate in vivo that APP-induced disruption of axonal transport can be rescued by dominant-negative Rab5, we turned to a transgenic Drosophila model that expresses human APP695 (94). Previous studies have demonstrated that these flies have significant deficits in axonal transport due to APP-induced axonal blockages consisting of synaptic vesicles among other organelles (95) (see Supplmental Figure 4, A and B). Segmental immunostaining of nerve axons in third instar larvae with an antibody against cysteine string protein (CSP), a synaptic vesicle marker, was used to assess axonal blockages (96). The APP695 line had an average of $107 \pm 11.44$ blockades per larva as compared with $2 \pm 0.447$ blocks per larva in the ApplGAL4 line (Figure 12B, top row, and Figure 12C). We then performed crosses between the APP695 line and a strain expressing YFPRab5 ${ }^{\mathrm{WT}}$, YFP-Rab5 ${ }^{\mathrm{CA}}$, YFP-Rab5 ${ }^{\mathrm{DN}}$, or mCD8-GFP (Figure 12A). The pan-neuronal driver UAS was used to drive the expression of all the transgenes used in the study. We chose mCD8-GFP as a control for the effect of protein overexpression because it is similar in size to YFP-Rab5. The average number of axonal blocks per larva was $18.40 \pm 2.11$ for $\mathrm{mCD} 8-\mathrm{GFP}, 16.80 \pm 5.142$ for YFP-Rab5 ${ }^{\mathrm{WT}}(P=0.781), 34.60 \pm 11.49$ for YFP-Rab5 ${ }^{\mathrm{CA}}(P=$ $0.203)$, and $1.0 \pm 0.548$ for YFP-Rab5 ${ }^{\mathrm{DN}}(P<0.001)$ (Figure $12 \mathrm{~B}$, bottom two rows, and Figure 12D). Expression of mCD8-GFP or each of the Rab5 constructs alone did not induce axonal block- age (Supplemental Figure 4). However, when coexpressed with APP695, the dominant-negative form Rab5 ${ }^{\text {DN }}$, but not Rab5 ${ }^{\text {WT }}$ or Rab5 ${ }^{\mathrm{CA}}$, significantly reduced the presence of axonal blocks. Therefore, consistent with in vitro studies, these results support that APP acts, at least in part, through enhancing activation of Rab5 to induce deficits in axonal transport in vivo.

\section{Discussion}

Endolysosomal dysfunction is a characteristic of early neuropathological signature of AD and DS (24, 26, 34, 36, 37, 97). However, it is unclear whether and how such change contributes to neurodegeneration $(19,26,28,29)$. Here, we show that full-length APP and its $\beta$-CTF, acting through increased activation of Rab5, induce endosomal pathology with marked deficits in trafficking and signaling of NGF in BFCNs. Our study has revealed a mechanism by which APP and its products contribute to neurodegeneration, raising the possibility that endosomal dysfunction is an early event in neurodegeneration in $\mathrm{AD}$ and DS.

At the cellular level, endosomal dysfunction, i.e., the abnormal enlargement of $\mathrm{Rab5}^{+}$endosome, is present prior to the appearance of plaques and tangles in $\operatorname{AD}$ or DS $(26,29,97)$. We speculated that endosomal dysfunction might impact the trophic status of BFCNs. NGF is a target-derived neurotrophic factor that supports the survival, differentiation, and maintenance of BFCNs $(50,98)$. NGF signaling regulates expression of genes and cellular programs important for the BFCN phenotype, including cell size. Following endocytosis, the NGF/TrkA signaling complexes are trafficked to Rab5 $5^{+}$early endosomes. These "signaling endosomes" are then retrogradely transported to the corresponding cell bodies to transmit NGF trophic signals $(50,98)$. Thus, axonal trafficking mediated by $\mathrm{Rab}^{+}$endosomes plays a critical role in maintaining the trophic status of BFCNs, and alteration in these aspects could potentially disrupt axonal transport, resulting in neurodegenerative disorders $(88,99-107)$.

Rab5-GTP, in concert with tethering proteins and effectors, promotes homotypic fusion of endocytic vesicles and heterotypic fusion of endocytic and trans-Golgi network-derived vesicles with the early endosome, resulting in the enlargement and matura- 
A

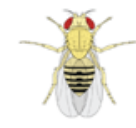

APP695 $x$

$\mid$

F1

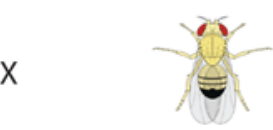

1. YFP-Rab5 $5^{\mathrm{WT}}$

2. YFP-Rab5 ${ }^{\mathrm{CA}}$

3. YFP-Rab5 ${ }^{\mathrm{DN}}$

4. mCD8-GFP

Quantitate \# of axonal blockages in 3rd instar larva with immunostaining for CSP

\section{B}
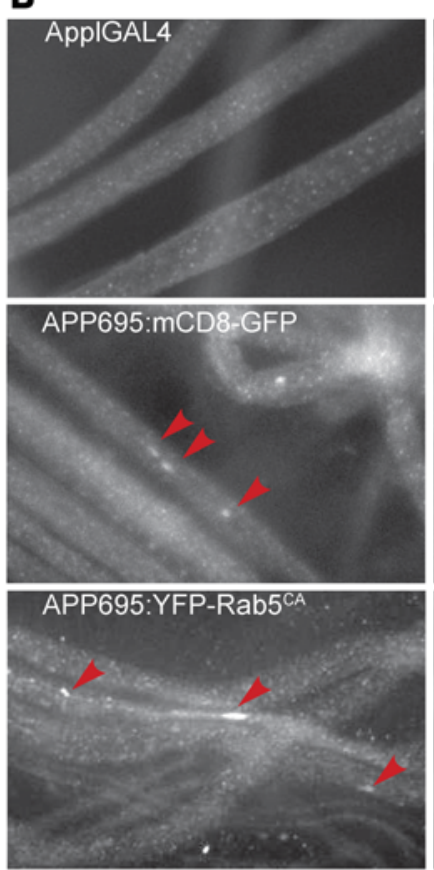
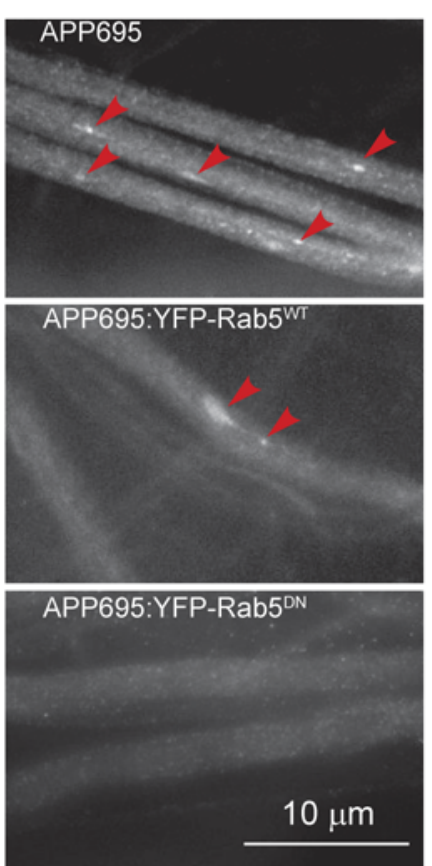

C

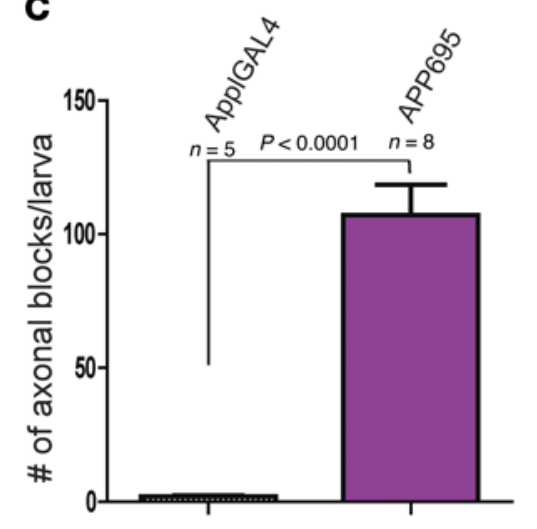

D

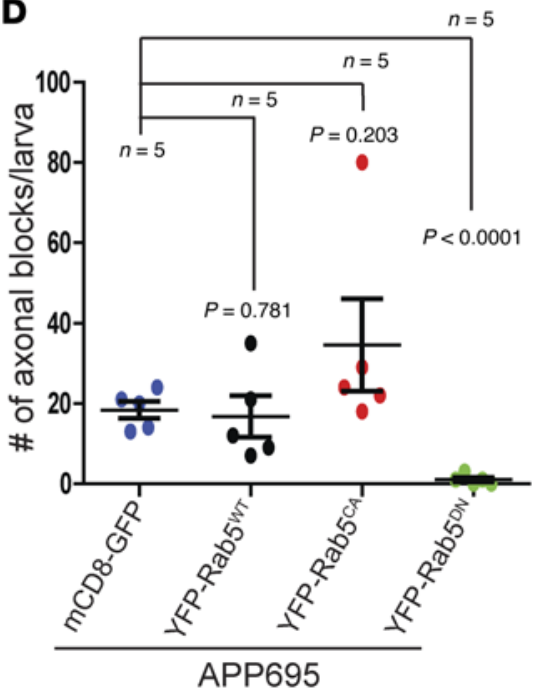

Figure 12. Expression of dominant-negative Rab5 suppressed APP-mediated axonal blockages in Drosophila larval segmental nerves. (A) Schematic of APP strain with stains that express different Rab5 constructs as well as mCD8 as the control. (B) Axonal blocks (arrowheads) in representative images of Drosophila third instar larval segmental nerves, immunostained with the synaptic vesicle marker cysteine string protein (CSP). Control (ApplCAL4) larvae show smooth CSP staining in larval segmental nerves, indicating no axonal transport defects (top left). Expression of human APP695 causes a severe axonal blockage phenotype (top right). The average number of axonal blocks/larva is shown in C. Coexpression of human APP695 with membrane-bound mCD8-GFP also shows axonal blockages in larval segmental nerves (B, middle left, arrowheads), which was used as a control for the effect of protein overexpression on axonal blockages. Representative images for larval segmental nerves resulted from the cross between human APP695 with wild-type YFP-Rab5 ${ }^{W T}$ (middle right), constitutively active YFP-Rab5 5A (bottom left), or dominant-negative YFP-Rab5 ${ }^{\mathrm{DN}}$ (bottom right). Axonal blockages (arrowheads) were quantitated within the entire larvae for each genotype, and the average number of axonal blocks for each genotype is shown in D. Expression of YFP-Rab5 ${ }^{\mathrm{DN}}$ with APP695 significantly reduced the amount of axonal blocks as compared with larvae expressing human APP695 with mCD8-GFP $(P<0.0001$, Student's $t$ test). Data represent mean \pm SEM. Scale bars: $10 \mu \mathrm{m}$.

tion of early endosomes $(22,23,38)$. Accordingly, one measure of the status of Rab5 activity is the size of early endosomes. Since enlargement of Rab5 ${ }^{+}$endosomes has been well established in DS, we asked might be the impact of increased levels of APP and its products. We found that increased expression of APP or its $\beta$-CTF led to hyperactivation of Rab5 and induced abnormal enlargement of the Rab5 ${ }^{+} \mathrm{NGF}$ signaling endosomes, likely impairing trophic delivery via axonal transport.

Although the mechanism(s) by which increased levels of fulllength APP and its $\beta$-CTF result in increased Rab5 activity are unknown, a Rab5 guanidine nucleotide exchange factor (GEF) such as Rabex-5 - shown herein to produce the same effects as APP overexpression - could play a role. Interestingly, RIN3, another Rab5 GEF $(108,109)$, has been identified in a large-scale genome-wide association study of AD (110). Recruitment of such effectors $(19,111,112)$ to endosomes would increase the level of Rab5 on these organelles.

There are at least two scenarios for explaining how increased activation of Rab5 impairs neuronal trophic signaling. The abnormally enlarged Rab5+ endosomes that carry NGF signals may experience greater difficulty in moving retrogradely within the axon, resulting in a net decrease in the signals delivered to the soma, eventually leading to a deficiency in trophic support for BFCNs. Increased Rab5 activation would also promote premature delivery of NGF signals to late endosomes/lysosomes, causing premature degradation of the NGF/TrkA signals (56), 
which would also decrease trophic support for BFCNs. There may be other mechanisms by which transport kinetics or the stoichiometry of motor proteins (including kinesins) are altered on NGF signaling endosomes. For instance, the C-terminal domain of APP directly (113) or indirectly (114) binds to kinesins that may impact trafficking of NGF signals. APP can also potentially interact with TrkA to influence their trafficking and signaling, as demonstrated recently (75), for the Y682 residue of APP is apparently a target for TrkA (115). Yet it is unclear how the interaction between APP and TrkA or phosphorylation of APP by TrkA is impacted in neurons under conditions where full-length APP and/or $\beta-\mathrm{CTF}$ is accumulated.

The adverse impact of $\beta$-CTF on synaptic plasticity and neuronal function has attracted increasing interest $(35,41-44,58)$. In our present studies, $\beta$-CTF, but not $\alpha$-CTF, had detrimental effects on neuronal function, although they only differ in the first $16 \mathrm{~N}$-terminal residues (see Methods). We speculate these 16 residues may play a role in mediating the adverse effect of $\beta$-CTF. Future studies will be needed to decipher the underlying molecular mechanisms. Furthermore, we demonstrated that a GSI, while inhibiting production of $A \beta$ species (13-15), induced marked accumulation of $\beta$-CTFs and increased the level of activated Rab5 (Figure $4 \mathrm{C}$ ), raising the possibility that excessive $\beta$-CTFs contributed to worsening cognitive function in AD patients $(13-15,40)$.

Endolyosomal changes predispose to other AD markers and pathogenetic events. The link with APP is obvious, especially in view of the trafficking of APP to the endosome and its processing therein, including the production of CTFs and the $A \beta$ peptide. The impact of mutations in APP and presenilin on APP processing and the early endosome are relevant to understanding how FAD creates endosomal pathology. The question of what links may exist between the endosome and plaque formation also deserves attention. Our study highlights the importance of endosomal function in regulating retrograde axonal trafficking and signaling of NGF and provides a mechanistic explanation whereby increased APP expression, via Rab5 activation, compromises retrograde transport of NGF in BFCNs. Future studies will be needed to determine whether reducing Rab5 activation will prevent or reverse neurodegeneration in AD and DS.

\section{Methods}

Chemicals, reagents, and antibodies. HBSS, Neurobasal, B27, GlutaMAX, Lipofectamine 2000, donkey anti-goat IgG-Alexa 568 conjugates, donkey anti-rabbit IgG-Alexa 488, and streptavidin-QD655 conjugates were from Invitrogen. DMEM high glucose was from Mediatech. FBS was from Phoenix Research Products. HEPES, polyL-lysine, cytosine- $\beta$-D-arabinofuranoside (AraC), and bisbenzimide H (Hoechst 33258) were from Sigma-Aldrich. BMS-299897 (GSI) was from by Steven L. Wagner (UCSD). Mouse purified NGF was as previously published (116). GTP agarose beads were from Innova Biosciences or Sigma-Aldrich.

Mouse anti-GFP IgGs (sc-9996), mouse anti-pErk1/2 (E6) IgGs (sc-271270), and rabbit anti-Rab5B IgGs (sc-598) were from Santa Cruz Biotechnology Inc.; rabbit anti-TrkA was from EMD Millipore (catalog 06-574). Mouse anti-GAPDH IgGs (GT239) were from GeneTex. Rabbit antibodies against pCREB(pSer133) (no. 9191), pErk1/2 (no. 9101), and total Erk1/2 (no. 9102) were from Cell Signaling Tech- nology. Goat anti-ChAT IgGs were from Millipore. Rabbit IgGs against the C-terminus of APP were from Steven L. Wagner. Goat anti-rabbit or anti-mouse IgG-HRP, biotin-SP-conjugated donkey anti-rabbit $\mathrm{IgG}$, and fluorescein-conjugated streptavidin were from Jackson ImmunoResearch Laboratories Inc.

Plasmids and siRNAs. The C99-GFP construct (117) was used to create the C83 and AICD-GFP construct (57). The C83 vector was made by deleting from $\mathrm{C} 99$ only the codons for the first 16 amino acid residues (bold), but not the 19-residue secretory signal sequence human APP (underlined) (sequence ID: gb|AAC13654.1|): C99-GFP 1, MLPGLALLLLAAWTARALEDAEFRHDSGYEVHHQKLVFFAEDVGSNKGAI . . . C83 lacks only the 16 residues (bold) of the human $\beta$-amyloid peptides (sequence ID: gb|AAB28602.1|) from the C99 construct: C83-GFP 1 MLPGLALLLLAAWTARALE-LVFFAED-

VGSNKGAI... The AICD-GFP construct was made by deleting the $\mathrm{A} \beta_{42}$ sequence from C99, resulting in: M(T)VIVITLVMLKKKQYTSIHHG ... ( $\mathrm{T}$ was changed to $\mathrm{M}$ for the translational start).

The pcDNA3-APP-EGFP was previously described (118), and the $\mathrm{APP}^{\mathrm{M} 596 \mathrm{~V}-\mathrm{YFP}}$ and APP ${ }^{\mathrm{SWE}}$ constructs were as previously described (57). GFP-Rab5 ${ }^{\mathrm{WT}}$, GFP-Rab ${ }^{5 \mathrm{C} 79 \mathrm{~L}}$, and GFP-Rab5 ${ }^{\mathrm{S} 34 \mathrm{~N}}$ plasmids were from Marino Zerial (Max Planck Institute of Molecular Cell Biology and Genetics, Dresden, Germany). The mCherry-Rab5 ${ }^{\mathrm{s} 34 \mathrm{~N}}$, mCherryRab5 ${ }^{\mathrm{WT}}$, and APP-mCherry expression vectors were generated by subcloning the cDNAs into the pcDNA3-mCherry vector (56). A fulllength Rabex- 5 cDNA was generated by PCR using a human brain cDNA library and was cloned into the pEGFP-N1 vector between HindIII and SalI. All sequences were verified by sequencing. The N-TER Nanoparticle Transfection System (Sigma-Aldrich) was used for transfection of siRNA against rat APP (Sigma-Aldrich PDSIRNA2D: SASI_Rn01_00086595 starting at 366) or the MISSION siRNA Universal Negative Control \#1 (Sigma-Aldrich SIC001).

PC12M cell culture, transfection, and neurite outgrowth. PC12M cells were maintained and cultured (56). The cells were transfected using Lipofectamine 2000 (Invitrogen). For outgrowth assays, the medium was changed to DMEM containing $50 \mathrm{ng} / \mathrm{ml} \mathrm{NGF}$ for 48 hours following transfection. Neurites were measured and analyzed using ImageJ (http://imagej.nih.gov/ij/).

GTP-agarose pull-down assays and SDS-PAGE/immunoblotting. The amount of Rab5-GTP was assayed following a published protocol (56). Briefly, mouse brain tissues or cultured cells were lysed (50 mM Tris- $\mathrm{HCl} \mathrm{pH} \mathrm{7.5,} 250 \mathrm{mM} \mathrm{NaCl}, 5 \mathrm{mM} \mathrm{Mg}$ acetate, $0.5 \%$ Triton $\mathrm{X}-100$, and protease inhibitor), followed by centrifugation to produce supernatants. An aliquot of supernatants (15-20 $\mu \mathrm{l})$ was saved as the loading control. The supernatants were incubated with GTP-agarose beads overnight at $4^{\circ} \mathrm{C}$ with rotation. The beads were washed and boiled. The amount of Rab5-GTP was measured by SDSPAGE and blotted with an anti-Rab5 antibody.

Established protocols were followed for SDS-PAGE/immunoblotting $(105,119)$. Equal amounts of proteins were separated on 4-12\% NuPAGE Bis-Tris precast gels (Invitrogen) and then electrotransferred to PVDF membranes, which were blocked with $5 \%$ nonfat milk and probed with specific antibodies. All blots were captured using ChemiDoc XRS+ (Bio-Rad), and only blots within linear ranges were quantitated using ImageLab 3.0.1 software (Bio-Rad). All experiments were repeated $\geq 3$ times.

Primary BFCNs culture and transfection. BFCNs were cultured from rat E18 embryos or transgenic mouse E17 embryos as 
described previously (120). Briefly, basal forebrains were dissected, dissociated, and resuspended in Neurobasal with 10\% FBS, B27, GlutaMAX, and $50 \mathrm{ng} / \mathrm{ml} \mathrm{NGF}$ and plated for 4 hours. Maintenance medium (Neurobasal with B27, GlutaMAX, and $50 \mathrm{ng} / \mathrm{ml} \mathrm{NGF}$ ) was added to the cell culture and incubated for 24 hours before being replaced with antimitotic medium (maintenance medium plus $1 \mu \mathrm{M}$ $\mathrm{AraC}$ ) for 12 hours, followed by switching to maintenance medium. For transfection, $0.5 \mu \mathrm{g}$ DNA and Lipofectamine 2000 were used. An approximately 15\%-20\% transfection efficiency was achieved. Cells were imaged 24-48 hours after transfection.

Immunofluorescence staining of BFCNs. BFCNs, cultured on PLLcoated glass coverslips, were fixed at DIV7 in 4\% PFA, $4 \%$ glucose for 15 minutes at $37^{\circ} \mathrm{C}$ and were permeabilized with $0.15 \%$ Triton $\mathrm{X}-100$ in PBS for 10 minutes, followed by blocking with $3 \%$ donkey serum in PBS for 1 hour. Neurons were incubated with primary antibodies (1:100 for goat anti-ChAT) overnight at $4^{\circ} \mathrm{C}$, rinsed, and incubated with donkey anti-goat IgG-Alexa 568 conjugates (1:500) for 1 hour at room temperature. The samples were then incubated with the other diluted primary antibodies (1:100 for rabbit anti-TrkA and 1:100 for rabbit anti-Rab5B) overnight at $4^{\circ} \mathrm{C}$. The samples were rinsed and incubated with biotin-SP-conjugated donkey anti-rabbit $\operatorname{IgG}(1: 500)$ for 1 hour at room temperature. The samples were rinsed and incubated with fluorescein-conjugated streptavidin (1:500) for 1 hour at room temperature. Nuclei were stained with Hoechst 33258 $(0.1 \mu \mathrm{g} / \mathrm{ml})$. All images were collected with a Leica inverted microscope (DMI6000B) with a $\times 100$ oil objective lens.

Live cell imaging, axonal transport of $Q D-N G F$, and confocal microscopy. PC12M cells or BFCNs were observed using the Leica inverted microscope, and images were captured using a Rolera MGi Fast 1397 camera (QImaging) with a $\times 100$ oil immersion objective (HCX PL Fluotar, NA 1.3) (Figure 3, A-D, Figure 4, A-D, Figure 6, A and $\mathrm{D}$, and Figure $7, \mathrm{~A}-\mathrm{E})$ (89). The microscope was equipped with an environmental chamber $\left(5 \% \mathrm{CO}_{2}, 37^{\circ} \mathrm{C}\right)$. Immediately before imaging, maintenance medium was replaced by $\mathrm{CO}_{2}$-independent medium. Image J was used to measure the size of $\mathrm{Rab5}^{+}$puncta.

For imaging of axonal transport in BFCNs cultured in microfluidic chambers $(89,90)$, $0.1 \mathrm{nM}$ QD-NGF was added to the axonal compartment and incubated for 4 hours. Time-lapse images were taken at 1 frame/second for a total of 120 seconds. Analysis of transport were performed using ImageJ.

For confocal microscopy, samples were fixed in 4\% PFA for 15 minutes at room temperature. Samples were then stained with antibodies following standard protocols (ref. 56; Figure 1, A and B, Figure $2 \mathrm{G}$, Figure 5D, and Supplemental Figure 1, B-D) or were immediately mounted for transfected samples. The samples were examined by confocal microscopy (Leica TCS SPE) with a $\times 63$ oil objective lens. ImageJ was used to measure the size of Rab5 $5^{+}$puncta.

Drosophila genetics. The UAS human APP695 strain was from Renato Paro (ETH Zurich, Zurich, Switzerland) (94), and the UASYFP-Rab5.WT, UAS-YFP-Rab5.CA, UAS-YFP-Rab5.DN, and UAS-mCD8-GFP stocks were from the Bloomington Drosophila Stock Center. Expression of human APP695 with YFP-Rab5.WT, -CA, or -DN was achieved by crossing these UAS lines to the panneuronal GAL4 driver Appl-GAL4 (95). Male UAS human APP695 and Rab5WT, -CA, and -DN or mDC8-GFP were first crossed to APPL-GAL4;B3/Pin virgin females. Males that were ApplGAL4/Y; APP695 or YFP-Rab5 (WT, CA, DN)/B3 were crossed to YFP-Rab5
(WT, CA, DN), APP695, or mCD8-GFP males, and the female larvae that were non-tubby were dissected for analysis. Tubby female siblings that were Appl-GAL4;YFP-Rab5 (WT, CA, DN)/+ or ApplGal4;mCD8-GFP/+ were used as controls.

Larval preparations, immunofluorescence, and quantification. Third instar larvae were dissected and fixed, and segmental nerve immunostaining was performed as described previously (96). Briefly, larvae were dissected in dissection buffer $(2 \times$ stock containing $128 \mathrm{mM} \mathrm{NaCl}$, $4 \mathrm{mM} \mathrm{MgCl}_{2}, 2 \mathrm{mM} \mathrm{KCl}, 5 \mathrm{mM}$ HEPES, and $36 \mathrm{mM}$ sucrose, $\mathrm{pH}$ 7.2), fixed in $4 \%$ formaldehyde, and incubated overnight with anti-CSP (1:10, Developmental Studies Hybridoma Bank at the University of Iowa). Following incubation in secondary antibodies (Alexa Fluor 568anti-mouse, A-11004, 1:100, Invitrogen), larvae were mounted using VECTASHIELD mounting medium (Vector Laboratories). Images were collected using a Nikon T2200 fluorescence microscope and analyzed with MetaMorph (Molecular Devices) (95, 96). Quantification of blockages was carried out on collected images using ImageJ as described previously (96). For each genotype, images were collected from 5-9 larvae along their entire body length. Using the threshold, density slice, and particle analysis functions in ImageJ, we quantified and analyzed axonal blockages as described previously (95).

Statistics. All experiments were repeated at least 3 times independently. To quantitate the size of endosomes, we counted 20-30 cells each time. Data represent mean \pm SEM. Statistical analyses and calculation of $P$ values were performed using Prism5 (GraphPad Software); Student's $t$ test was used for pairwise comparisons and 1-way ANOVA for multiple comparisons. $P$ values less than 0.05 were considered statistically significant, and $P$ values less than 0.01 were considered statistically highly significant.

Study approval. All experimental protocols involving the use of animals were approved by the IACUC of UCSD.

\section{Author contributions}

WX, AMW, JAW, FF, XW, YW, MLP, XZ, MS, and CW performed the experiments. SC, SG, JD, WCM, and CW conceived the experimental deigns. WX, JD, WCM, and CW analyzed the data and wrote the manuscript.

\section{Acknowledgments}

We thank Pauline Yue Hu for technical support. The study is support by grants from the NIH (PN2EY016525), LuMind Research Down Syndrome Foundation, Larry L. Hillblom Foundation, Alzheimer's Association, Thrasher Research Fund, and Tau Consortium to W.C. Mobley and C. Wu; the NIH (NS084386 and NS092024) to S. Gunawardena; and the Ministry of Science and Technology of the People's Republic of China (2014CB965002, 2012BAI10B03), National Natural Science Foundation of China (81171200), Science and Technology Commission of Shanghai Municipality (13JC1401502, 13140904000), and Shanghai Municipal Education Commission (12ZZ115) to J. Ding, W. Xu, Y. Wu.

Address correspondence to: Jianqing Ding, Shanghai Jiaotong University School of Medicine Ruijin Hospital, Institute of Neurology, 197 Ruijin Er Rd., Building \#35, Room \#404, Shanghai 200025, China. Phone: 011.86.216.445.7249; E-mail: jqding18@163.com. Or to: William C. Mobley, Department of Neurosciences, University of California, San Diego, CNCB Building, Room 100, 9500 
Gilman Drive, La Jolla, California 92093-0752, USA. Phone: 858.534.9434; E-mail: wmobley@ucsd.edu. Or to: Chengbiao $\mathrm{Wu}$, Department of Neurosciences, University of California, San
Diego, Medical Teaching Facility, Room 312 MC-0624, 9500 Gilman Drive, La Jolla, California 92093-0624, USA. Phone: 858.534.0996; E-mail: chw049@ucsd.edu.
1. Selkoe DJ. The cell biology of $\beta$-amyloid precursor protein and presenilin in Alzheimer's disease. Trends Cell Biol. 1998;8(11):447-453.

2. Selkoe DJ. Toward a comprehensive theory for Alzheimer's disease. Ann N Y Acad Sci. 2000;924:17-25.

3. Selkoe DJ. Alzheimer's disease: genes, proteins, and therapy. Physiol Rev. 2001;81(2):741-766.

4. Holtzman DM, Mandelkow E, Selkoe DJ. Alzheimer disease in 2020. Cold Spring Harb Perspect Med. 2012;2(11):a011585.

5. Selkoe DJ. Alzheimer's disease. Cold Spring Harb Perspect Biol. 2011;3(7):a004457.

6. Selkoe DJ. Aging, amyloid, and Alzheimer's disease: a perspective in honor of Carl Cotman. Neurochem Res. 2003;28(11):1705-1713.

7. Mori C, et al. Intraneuronal A $\beta 42$ accumulation in Down syndrome brain. Amyloid. 2002;9(2):88-102.

8. Hardy J, Selkoe DJ. The amyloid hypothesis of Alzheimer's disease: progress and problems on the road to therapeutics. Science. 2002;297(5580):353-356.

9. Cummings BJ, et al. Diffuse plaques contain Cterminal $\mathrm{A} \beta 42$ and not $\mathrm{A} \beta 40$ : evidence from cats and dogs. Neurobiol Aging. 1996;17(4):653-659.

10. Citron M, et al. Excessive production of amyloid beta-protein by peripheral cells of symptomatic and presymptomatic patients carrying the Swedish familial Alzheimer disease mutation. Proc Natl Acad Sci U S A . 1994;91(25):11993-11997.

11. Cork LC, et al. Neurofibrillary tangles and senile plaques in aged bears. JNeuropathol Exp Neurol. 1988;47(6):629-641.

12. Rasool CG, Svendsen CN, Selkoe DJ. Neurofibrillary degeneration of cholinergic and noncholinergic neurons of the basal forebrain in Alzheimer's disease. Ann Neurol. 1986;20(4):482-488.

13. Coric V, et al. Safety and tolerability of the $\gamma$-secretase inhibitor avagacestat in a phase 2 study of mild to moderate Alzheimer disease. Arch Neurol. 2012;69(11):1430-1440.

14. Doody RS, Aisen PS, Iwatsubo T. Semagacestat for treatment of Alzheimer's disease. N EnglJMed. 2013;369(17):1661.

15. Doody RS, et al. A phase 3 trial of semagacestat for treatment of Alzheimer's disease. N Engl JMed. 2013;369(4):341-350.

16. Donohue MC, et al. The preclinical Alzheimer cognitive composite: measuring amyloid-related decline. JAMA Neurol. 2014;71(8):961-970.

17. Ginsberg SD, et al. Regional selectivity of rab5 and rab7 protein upregulation in mild cognitive impairment and Alzheimer's disease. JAlzheimers Dis. 2010;22(2):631-639.

18. Grbovic OM, et al. Rab5-stimulated up-regulation of the endocytic pathway increases intracellular beta-cleaved amyloid precursor protein carboxylterminal fragment levels and $A \beta$ production. JBiol Chem. 2003;278(33):31261-31268.

19. Laifenfeld D, et al. Rab5 mediates an amyloid precursor protein signaling pathway that leads to apoptosis. J Neurosci. 2007;27(27):7141-7153.

20. Ikin AF, et al. Alzheimer amyloid protein precursor is localized in nerve terminal preparations to Rab5containing vesicular organelles distinct from those implicated in the synaptic vesicle pathway. J Biol Chem. 1996;271(50):31783-31786.

21. Buxbaum JD, Greengard P. Regulation of APP processing by intra- and intercellular signals. Ann N Y Acad Sci. 1996;777:327-331.

22. Bucci $C$, et al. The small GTPase rab5 functions as a regulatory factor in the early endocytic pathway. Cell. 1992;70(5):715-728.

23. Gorvel JP, et al. rab5 controls early endosome fusion in vitro. Cell. 1991;64(5):915-925.

24. Cataldo AM, et al. Endocytic pathway abnormalities precede amyloid beta deposition in sporadic Alzheimer's disease and Down syndrome: differential effects of APOE genotype and presenilin mutations. Am J Pathol. 2000;157(1):277-286.

25. Ihara Y, Morishima-Kawashima M, Nixon R. The ubiquitin-proteasome system and the autophagic-lysosomal system in Alzheimer disease. Cold Spring Harb Perspect Med. 2012;2(8):a006361.

26. Cataldo AM, et al. App gene dosage modulates endosomal abnormalities of Alzheimer's disease in a segmental trisomy 16 mouse model of down syndrome. J Neurosci. 2003;23(17):6788-6792.

27. Cattaneo A, Calissano P. Nerve growth factor and Alzheimer's disease: new facts for an old hypothesis. Mol Neurobiol. 2012;46(3):588-604.

28. Salehi A, et al. Increased App expression in a mouse model of Down's syndrome disrupts NGF transport and causes cholinergic neuron degeneration. Neuron. 2006;51(1):29-42.

29. Ginsberg SD, et al. Microarray analysis of hippocampal CA1 neurons implicates early endosomal dysfunction during Alzheimer's disease progression. Biol Psychiatry. 2010;68(10):885-893.

30. Golde TE, et al. Processing of the amyloid protein precursor to potentially amyloidogenic derivatives. Science. 1992;255(5045):728-730.

31. Selkoe DJ, et al. $\beta$-Amyloid precursor protein of Alzheimer disease occurs as 110- to 135-kilodalton membrane-associated proteins in neural and nonneural tissues. Proc Natl Acad Sci U S A. 1988;85(19):7341-7345

32. Vetrivel KS, et al. Alzheimer disease Abeta production in the absence of S-palmitoylation-dependent targeting of BACE1 to lipid rafts. J Biol Chem. 2009;284(6):3793-3803.

33. Thinakaran G, Koo EH. Amyloid precursor protein trafficking, processing, and function. J Biol Chem. 2008;283(44):29615-29619.

34. Guo Q, et al. APP physiological and pathophysiological functions: insights from animal models. Cell Res. 2012;22(1):78-89.

35. Kozlowski MR, et al. The neurotoxic carboxyterminal fragment of the Alzheimer amyloid precursor binds specifically to a neuronal cell surface molecule: $\mathrm{pH}$ dependence of the neurotoxicity and the binding. J Neurosci. 1992;12(5):1679-1687.
36. Jiang $Y$, et al. Alzheimer's-related endosome dysfunction in Down syndrome is $A \beta$-independent but requires APP and is reversed by BACE-1 inhibition. Proc Natl Acad Sci U S A. 2010;107(4):1630-1635.

37. Nosheny RL, et al. Increased cortical synaptic activation of TrkB and downstream signaling markers in a mouse model of Down Syndrome. Neurobiol Dis. 2015;77:173-190.

38. Cooper JD, et al. Failed retrograde transport of NGF in a mouse model of Down's syndrome: reversal of cholinergic neurodegenerative phenotypes following NGF infusion. Proc Natl Acad Sci US A. 2001;98(18):10439-10444.

39. Albright CF, et al. Pharmacodynamics of selective inhibition of gamma-secretase by avagacestat. JPharmacol Exp Ther. 2013;344(3):686-695.

40. Weissmiller AM, et al. A gamma-secretase inhibitor, but not a $\gamma$-secretase modulator, induced defects in BDNF axonal trafficking and signaling: evidence for a role for APP. PLoS One. 2015;10(2):e0118379.

41. Mitani Y, et al. Differential effects between gamma-secretase inhibitors and modulators on cognitive function in amyloid precursor protein-transgenic and nontransgenic mice. JNeurosci. 2012;32(6):2037-2050.

42. Lauritzen I, et al. The $\beta$-secretase-derived C-terminal fragment of $\beta A P P, C 99$, but not $A \beta$, is a key contributor to early intraneuronal lesions in triple-transgenic mouse hippocampus. JNeurosci. 2012;32(46):16243-16255a

43. Bittner T, et al. $\gamma$-Secretase inhibition reduces spine density in vivo via an amyloid precursor protein-dependent pathway. J Neurosci. 2009;29(33):10405-10409.

44. Tamayev R, et al. $\beta$ - but not $\gamma$-secretase proteolysis of APP causes synaptic and memory deficits in a mouse model of dementia. EMBO Mol Med. 2012;4(3):171-179.

45. Chao MV. Neurotrophins and their receptors: a convergence point for many signalling pathways. Nat Rev Neurosci. 2003;4(4):299-309.

46. Ginty DD, Segal RA. Retrograde neurotrophin signaling: Trk-ing along the axon. Curr Opin Neurobiol. 2002;12(3):268-274

47. Glebova NO, Ginty DD. Growth and survival signals controlling sympathetic nervous system development. Annu Rev Neurosci. 2005;28:191-222.

48. Holtzman DM, et al. p140trk mRNA marks NGF-responsive forebrain neurons: evidence that trk gene expression is induced by NGF. Neuron. 1992;9(3):465-478.

49. Sofroniew MV, et al. Survival of adult basal forebrain cholinergic neurons after loss of target neurons. Science. 1990;247(4940):338-342.

50. Li Y, et al. Regulation of TrkA and ChAT expression in developing rat basal forebrain: evidence that both exogenous and endogenous NGF regulate differentiation of cholinergic neurons. JNeurosci. 1995;15(4):2888-2905.

51. Holtzman DM, et al. TrkA expression in the CNS: evidence for the existence of several 
novel NGF-responsive CNS neurons. J Neurosci. 1995;15(2):1567-1576.

52. Perry EK. Nerve growth factor and the basal forebrain cholinergic system: a link in the etiopathology of neurodegenerative dementias? Alzheimer Dis Assoc Disord. 1990;4(1):1-13.

53. Reeves RH, et al. A mouse model for Down syndrome exhibits learning and behaviour deficits. Nat Genet. 1995;11(2):177-184.

54. Simonsen A, et al. EEA1 links PI(3)K function to Rab5 regulation of endosome fusion. Nature. 1998;394(6692):494-498.

55. Stenmark H, et al. Inhibition of rab5 GTPase activity stimulates membrane fusion in endocytosis. EMBO J.1994;13(6):1287-1296.

56. Zhang K, et al. Defective axonal transport of Rab7 GTPase results in dysregulated trophic signaling. JNeurosci. 2013;33(17):7451-7462.

57. Rodrigues EM, Weissmiller AM, Goldstein LS. Enhanced beta-secretase processing alters APP axonal transport and leads to axonal defects. Hum Mol Genet. 2012;21(21):4587-4601.

58. Bryan-Sisneros AA, et al. Toxic effect of the $\beta$-amyloid precursor protein $\mathrm{C}$-terminus fragment and $\mathrm{Na}^{+} / \mathrm{Ca}^{2+}$ gradients. Neuroreport. 2000;11(15):3357-3360

59. Hartell NA, Suh YH. Peptide fragments of $\beta$-amyloid precursor protein: effects on parallel fiber-Purkinje cell synaptic transmission in rat cerebellum. J Neurochem. 2000;74(3):1112-1121.

60. Oster-Granite ML, et al. Age-dependent neuronal and synaptic degeneration in mice transgenic for the $\mathrm{C}$ terminus of the amyloid precursor protein. J Neurosci. 1996;16(21):6732-6741.

61. Horiuchi H, et al. A novel Rab5 GDP/GTP exchange factor complexed to Rabaptin-5 links nucleotide exchange to effector recruitment and function. Cell.1997;90(6):1149-1159.

62. Sanford JC, Pan Y, Wessling-Resnick M. Prenylation of Rab5 is dependent on guanine nucleotide binding. J Biol Chem. 1993;268(32):23773-23776.

63. Anderson JJ, Holtz G, Baskin PP, et al. Reductions in $\beta$-amyloid concentrations in vivo by the $\gamma$-secretase inhibitors BMS-289948 and BMS-299897. Biochem Pharmacol. 2005;69(4):689-698.

64. Borgegard T, et al. First and second generation gamma-secretase modulators (GSMs) modulate $\mathrm{A} \beta$ production through different mechanisms. J Biol Chem. 2012;287(15):11810-11819.

65. Crump CJ, Johnson DS, Li YM. Development and mechanism of $\gamma$-secretase modulators for Alzheimer's disease. Biochemistry. 2013;52(19):3197-3216.

66. Grosveld GC. $\gamma$-Secretase inhibitors: Notch so bad. Nat Med. 2009;15(1):20-21.

67. Holtzman DM, Mandelkow E, Selkoe DJ. Alzheimer disease in 2020. Cold Spring Harb Perspect Med. 2012;2(11):a011585.

68. Kopan R, Ilagan MXG. $\gamma$-Secretase: proteasome of the membrane? Nat Rev Mol Cell Biol. 2004;5(6):499-504.

69. Selkoe D, Kopan R. Notch and Presenilin: regulated intramembrane proteolysis links development and degeneration. Annu Rev Neurosci. 2003;26:565-597.

70. Wolfe MS. $\gamma$-Secretase inhibitors and modulators for Alzheimer's disease. J Neurochem. 2012;120(suppl 1):89-98.
71. Liu J, et al. Nerve growth factor-mediated neurite outgrowth via regulation of Rab5. Mol Biol Cell. 2007;18(4):1375-1384.

72. Zhang Y, et al. Cell surface Trk receptors mediate NGF-induced survival while internalized receptors regulate NGF-induced differentiation. J Neurosci. 2000;20(15):5671-5678.

73. Lee JP, et al. APP carboxyl-terminal fragment without or with abeta domain equally induces cytotoxicity in differentiated PC12 cells and cortical neurons. JNeurosci Res. 2000;60(4):565-570.

74. Sandhu FA, et al. Expression of the $\mathrm{C}$ terminus of the amyloid precursor protein alters growth factor responsiveness in stably transfected PC12 cells. Proc Natl Acad Sci U S A. 1996;93(5):2180-2185.

75. Zhang YW, et al. APP regulates NGF receptor trafficking and NGF-mediated neuronal differentiation and survival. PLoS One. 2013;8(11):e80571.

76. Gibbs RB. Impairment of basal forebrain cholinergic neurons associated with aging and long-term loss of ovarian function. Exp Neurol. 1998;151(2):289-302.

77. Granholm AC, Sanders LA, Crnic LS. Loss of cholinergic phenotype in basal forebrain coincides with cognitive decline in a mouse model of Down's syndrome. Exp Neurol. 2000;161(2):647-663.

78. Mufson EJ, Bothwell M, Kordower JH. Loss of nerve growth factor receptor-containing neurons in Alzheimer's disease: a quantitative analysis across subregions of the basal forebrain. Exp Neurol. 1989;105(3):221-232.

79. Mufson EJ, et al. Human cholinergic basal forebrain: chemoanatomy and neurologic dysfunction. JChem Neuroanat. 2003;26(4):233-242.

80. Mufson EJ, et al. Decreased trkA gene expression within basal forebrain neurons in Alzheimer's disease. Neuroreport. 1996;8(1):25-29.

81. Mufson EJ, et al. Loss of basal forebrain P75(NTR) immunoreactivity in subjects with mild cognitive impairment and Alzheimer's disease. J Comp Neurol. 2002;443(2):136-153.

82. Muth K, et al. Mild cognitive impairment in the elderly is associated with volume loss of the cholinergic basal forebrain region. Biol Psychiatry. 2010;67(6):588-591.

83. Cabeza C, et al. Cholinergic abnormalities, endosomal alterations and up-regulation of nerve growth factor signaling in Niemann-Pick type C disease. Mol Neurodegener. 2012;7:11.

84. Williams B, Granholm AC, Sambamurti K. Age-dependent loss of NGF signaling in the rat basal forebrain is due to disrupted MAPK activation. Neurosci Lett. 2007;413(2):110-114.

85. Ginsberg SD, et al. Down regulation of trk but not p75NTR gene expression in single cholinergic basal forebrain neurons mark the progression of Alzheimer's disease. J Neurochem. 2006;97(2):475-487.

86. Yuen EC, et al. Nerve growth factor and the neurotrophic factor hypothesis. Brain Dev. 1996;18(5):362-368.

87. Das U, et al. Activity-induced convergence of APP and BACE-1 in acidic microdomains via an endocytosis-dependent pathway. Neuron. 2013;79(3):447-460.
88. Wu C, et al. The coming of age of axonal neurotrophin signaling endosomes. J Proteomics. 2009;72(1):46-55.

89. Cui B, et al. One at a time, live tracking of NGF axonal transport using quantum dots. Proc Natl Acad Sci U S A. 2007;104(34):13666-13671.

90. Sung K, et al. A novel method for producing mono-biotinylated, biologically active neurotrophic factors: an essential reagent for single molecule study of axonal transport. JNeurosci Methods. 2011;200(2):121-128.

91. Zhao X, Zhou Y, Weissmiller AM, Pearn ML, Mobley WC, Wu C. Real-time imaging of axonal transport of quantum dot-labeled BDNF in primary neurons. J Vis Exp. 2014;(91):51899.

92. Suo D, et al. Coronin-1 is a neurotrophin endosomal effector that is required for developmental competition for survival. Nat Neurosci. 2014;17(1):36-45.

93. Levi-Montalcini R, Hamburger V. Selective growth stimulating effects of mouse sarcoma on the sensory and sympathetic nervous system of the chick embryo. J Exp Zool. 1951;116(2):321-361.

94. Fossgreen A, et al. Transgenic Drosophila expressing human amyloid precursor protein show gamma-secretase activity and a blistered-wing phenotype. Proc Natl Acad Sci U S A. 1998;95(23):13703-13708.

95. Gunawardena S, Goldstein LS. Disruption of axonal transport and neuronal viability by amyloid precursor protein mutations in Drosophila. Neuron. 2001;32(3):389-401.

96. Fye $\mathrm{S}$, et al. Visualization of larval segmental nerves in 3(rd) instar Drosophila larval preparations. JVis Exp. 2010;(43):2128.

97. Israel MA, et al. Probing sporadic and familial Alzheimer's disease using induced pluripotent stem cells. Nature. 2012;482(7384):216-220.

98. Sofroniew MV, Howe CL, Mobley WC. Nerve growth factor signaling, neuroprotection, and neural repair. Аnnu Rev Neurosci. 2001;24:1217-1281.

99. Goldstein LS. Axonal transport and neurodegenerative disease: can we see the elephant? Prog Neurobiol. 2012;99(3):186-190.

100.Kanaan NM, et al. Axonal degeneration in Alzheimer's disease: when signaling abnormalities meet the axonal transport system. Exp Neurol. 2013;246:44-53.

101.Ye X, Tai W, Zhang D. The early events of Alzheimer's disease pathology: from mitochondrial dysfunction to BDNF axonal transport deficits. Neurobiol Aging. 2012;33(6):1122.

102.Strom AL, et al. Retrograde axonal transport and motor neuron disease. J Neurochem. 2008;106(2):495-505.

103.Schindowski K, Belarbi K, Buee L. Neurotrophic factors in Alzheimer's disease: role of axonal transport. Genes Brain Behav. 2008;7(suppl 1):43-56.

104.El-Kadi AM, Soura V, Hafezparast M. Defective axonal transport in motor neuron disease. J Neurosci Res. 2007;85(12):2557-2566.

105. Baloh RH, et al. Altered axonal mitochondrial transport in the pathogenesis of Charcot-Marie-Tooth disease from mitofusin 2 mutations. JNeurosci. 2007;27(2):422-430.

106.Stokin GB, Goldstein LS. Axonal transport 
and Alzheimer's disease. Annu Rev Biochem. 2006;75:607-627.

107. Charrin BC, Saudou F, Humbert S. Axonal transport failure in neurodegenerative disorders: the case of Huntington's disease. Pathol Biol (Paris). 2005;53(4):189-192.

108. Kajiho H, et al. RIN3: a novel Rab5 GEF interacting with amphiphysin II involved in the early endocytic pathway. JCell Sci. 2003;116(pt 20):4159-4168.

109. Kajiho H, et al. Characterization of RIN3 as a guanine nucleotide exchange factor for the Rab5 subfamily GTPase Rab31. J Biol Chem. 2011;286(27):24364-24373.

110. Lambert JC, et al. Meta-analysis of 74,046 individuals identifies 11 new susceptibility loci for Alzheimer's disease. Nat Genet. 2013;45(12):1452-1458.

111. Chen Y, et al. APP-BP1 inhibits A $\beta 42$ levels by interacting with Presenilin-1. Mol Neurodegener. 2007;2:3.

112. Chen Y, et al. APP-BP1 mediates APP-induced apoptosis and DNA synthesis and is increased in Alzheimer's disease brain. J Cell Biol. 2003;163(1):27-33.

113. Kamal A, et al. Axonal transport of amyloid precursor protein is mediated by direct binding to the kinesin light chain subunit of kinesin-I. Neuron. 2000;28(2):449-459.

114. Inomata $\mathrm{H}$, et al. A scaffold protein JIP-1b enhances amyloid precursor protein phosphorylation by JNK and its association with kinesin light chain 1. J Biol Chem. 2003;278(25):22946-22955.

115. Matrone C, et al. APP is phosphorylated by TrkA and regulates NGF/TrkA signaling. J Neurosci. 2011;31(33):11756-11761.

116. Mobley WC, et al. Nerve growth factor increases choline acetyltransferase activity in developing basal forebrain neurons. Brain Res. 1986;387(1):53-62.

117. Kaether C, et al. Amyloid precursor protein and Notch intracellular domains are generated after transport of their precursors to the cell surface. Traffic. 2006;7(4):408-415.

118. Fjorback AW, et al. Retromer binds the FANSHY sorting motif in SorLA to regulate amyloid precursor protein sorting and processing. J Neurosci. 2012;32(4):1467-1480.

119. Wu C, Lai CF, Mobley WC. Nerve growth factor activates persistent Rap1 signaling in endosomes. J Neurosci. 2001;21(15):5406-5416.

120. Schnitzler AC, Lopez-Coviella I, Blusztajn JK. Purification and culture of nerve growth factor receptor (p75)-expressing basal forebrain cholinergic neurons. Nat Protoc. 2008;3(1):34-40. 